\title{
Pacific
}

Journal of

Mathematics

APPLICATIONS OF LOOP GROUPS AND STANDARD MODULES TO JACOBIANS AND THETA FUNCTIONS OF ISOSPECTRAL CURVES

WILLI SCHWARZ 


\section{APPLICATIONS OF LOOP GROUPS AND STANDARD MODULES TO JACOBIANS AND THETA FUNCTIONS OF ISOSPECTRAL CURVES}

\section{WILLI SCHWARZ}

Let $L(z)$ be an element of $M_{n}\left(\mathbb{C}\left[z, z^{-1}\right]\right)$. In this work we study the structure of isospectral curves given by $f(z, \lambda)=0$, $f(z, \lambda)=\operatorname{det}(L(z)-\lambda)$, their Jacobians and the relationship between standard modules and the corresponding theta functions. We assume that $f(z, \lambda)$ is irreducible and nonsingular for $f(z, \lambda)=0$ and $z \in \mathbb{C}^{*}$.

The element $L(z)$ will be called good, if the centralizers $\mathfrak{C}_{ \pm}(L)$ of $L(z)$ in $M_{n}(\mathbb{C}[z])$ (resp. $M_{n}\left(\mathbb{C}\left[z^{-1}\right]\right)$ ) are the integral closure of $\mathbb{C}\left[z, z^{p} L\right]$ (resp. $M_{n}\left(\mathbb{C}\left[z^{-1}, z^{-q} L\right]\right)$ ) in $M_{n}\left(\mathbb{C}\left[z, z^{-1}\right]\right)$. The class of curves we analyze include nonsingular curves and the isospectral curve of the periodic Toda lattice. The latter curve is represented by a "tridiagonal" matrix $L(z)$.

The Jacobian variety is expressed as a quotient of certain centralizers of $L(z)$ which are computed in a completion $M_{n}\left(A_{w}\right)$ of $M_{n}\left(\mathbb{C}\left[z, z^{-1}\right]\right)$. If we assume further that $L(z)$ is an element of $\underline{S L}_{n}\left(\mathbb{C}\left[z, z^{-1}\right]\right)$ then the basic module of the universal central extension $\widehat{S L}_{n}\left(A_{w}\right)$ of $S L_{n}\left(A_{w}\right)$ is employed to define a function $\Theta$. This function $\Theta$ is defined in terms of representative functions on the "Lie theoretic" Jacobian and satisfies the functional equation of theta functions.

\section{Introduction.}

The relationship between completely integrable Hamiltonian systems, KacMoody Lie algebras and curve theory were studied systematically by $M$. Adler and P. van Moerbeke in [1], [2]. The main idea of their method is to associate to such a Hamiltonian system a Lax matrix differential equation of the form

$$
\frac{d L}{d t}=\left[L, M(L)^{+}\right]=\left[L, M(L)^{-}\right]
$$

where $L$ is an element of a loop algebra $\underline{\tilde{g}}=\underline{g} \otimes \mathbb{C}\left[z, z^{-1}\right]$ and $M(L)$ is a function of $L$. The associated isospectral curve $X_{L}$ is obtained as projective completion of the quasi-affine curve

$$
X^{a}=\left\{(z, \lambda) \in \mathbb{C}^{*} \times \mathbb{C} \mid \operatorname{det}(L(z)-\lambda)=0\right\} .
$$


The corresponding Lax equation of a completely integrable Hamiltonian system becomes linear on the Jacobian variety of the curve $X_{L}$. For those systems which are of relevance in physics, such as the periodic Toda system, the Euler-Arnold spinning top and the Neumann problem, the isospectral curves are hyperelliptic.

Certain classical integrable Hamiltonian systems which arise in mechanics or geometry, for instance the motion of a particle on an ellipsoid under a central force or the geodesic flow on an ellipsoid, were solved directly by J. Moser [15]. The solutions are expressed in terms of hyperelliptic theta functions on the Jacobian variety of the related hyperelliptic isospectral curve. The Jacobian variety is obtained as a quotient-space of an isospectral manifold. Its linear structure defined by the Hamiltonian vector fields of the integrals of the flow agrees with the linear structure as given by Abel's theorem. These ideas were developed further by D. Mumford in [18] to construct families of Jacobian varieties using dynamical systems.

Classical and quantum mechanical systems of Toda lattice type were studied in detail by R. Goodman and N. Wallach in a series of papers [6], [7], [8]. In the case of the generalized periodic Toda lattice the solution is calculated in terms of representative functions of standard modules of a Banach Lie group $\hat{G}_{w}$. The group $\hat{G}_{w}$ is obtained as a central extension of the connected and simply-connected loop group $\tilde{G}_{w}$ of the completion $\underline{\tilde{g}}_{w}$ of the Kac-Moody algebra $\tilde{g}$. To obtain explicit solutions the representative functions on standard modules defined by highest weight vectors are computed along certain one-parameter subgroups of $\hat{G}_{w}$. These functions define a system of non-linear ordinary differential equations. In the special case of $\widehat{S L}_{2}(\mathbb{R})$ this system of ordinary differential was solved explicitly and the solution described in terms of theta functions.

In this work we combine the Kac-Moody Lie algebra approach to Hamiltonian systems with the method of representative functions of standard modules to obtain our main results. The related Lax equation of an integrable Hamiltonian system

$$
\frac{d L}{d t}=\left[L, M(L)^{+}\right]=\left[L, M(L)^{-}\right]
$$

with $L \in M_{n}\left(\mathbb{C}\left[z, z^{-1}\right]\right)$ implies that $M(L)^{+}-M(L)^{-}$is contained in the centralizer of $L$ in $M_{n}\left(\mathbb{C}\left[z, z^{-1}\right]\right)$. Taking this fact into account we consider various centralizers of $L$ in a certain loop algebra and its associated loop group.

Suppose $L \in M_{n}\left(\mathbb{C}\left[z, z^{-1}\right]\right), L(z)=\sum_{j=-p}^{q} \ell_{j} z^{j}$ is such that its characteristic polynomial $f(z, \lambda)$ is irreducible and the isospectral curve $X_{L}$ is nonsingular for $z \in \mathbb{C}^{*}$. The calculation of centralizers will be done in a 
completion $M_{n}\left(A_{w}\right)$ of the loop algebra $M_{n}\left(\mathbb{C}\left[z, z^{-1}\right]\right)$. The completion is defined by the absolute convergence of weighted series of Fourier coefficients for certain weights $w$. These weights satisfy for some $\sigma, 1<\sigma<2$, the inequalities

$$
C_{t} \exp \left(t|k|^{1 / \sigma}\right) \leq w(k) \leq M_{\epsilon} \exp (\epsilon|k|)
$$

for all $t, \epsilon>0, k \in \mathbb{Z}$ and some positive constants $C_{t}, M_{\epsilon}$.

Let $\mathfrak{C}^{w}(L)$ denote the centralizer of $L$ in $M_{n}\left(A_{w}\right)$ and $\mathfrak{C}_{+}^{w}$ resp. $\mathfrak{C}_{-}^{w}$ the centralizers of $L$ in the respective completions of $M_{n}(\mathbb{C}[z])$ and $M_{n}\left(\mathbb{C}\left[z^{-1}\right]\right)$. We prove that if the curve $X_{L}$ is nonsingular or if $L$ is "tridiagonal" (i.e. $L$ is tridiagonal with an entry in the upper right corner and in the lower left corner) that the respective centralizers $\mathfrak{C}_{ \pm}(L)$ in $M_{n}(\mathbb{C}[z])$ resp. $M_{n}\left(\mathbb{C}\left[z^{-1}\right]\right)$ are the integral closure of $\mathbb{C}\left[z, z^{p} L\right]$ resp. of $\mathbb{C}\left[z^{-1}, z^{-q} L\right]$ in $M_{n}\left(\mathbb{C}\left[z, z^{-1}\right]\right)$. We consider $\mathbb{C}\left[z, z^{p} L\right]$ and $\mathbb{C}\left[z^{-1}, z^{-q} L\right]$ to be contained in $M_{n}\left(\mathbb{C}\left[z, z^{-1}\right]\right)$. The element $L \in M_{n}\left(\mathbb{C}\left[z, z^{-1}\right]\right)$ will be called good if it satisfies this additional condition.

If $C_{ \pm}^{w}(L)$ denote the analogue of the centralizers $\mathfrak{C}_{ \pm}^{w}(L)$ in the loop group $G L_{n}\left(A_{w}\right)$ we define

$$
\Lambda=\left\{V \in \mathfrak{C}^{w}(L) \mid e^{V} \in C_{-}^{w}(L) C_{+}^{w}(L)\right\} .
$$

We will prove that the Jacobian variety of the desingularized curve $X_{L}^{\prime}$, $\operatorname{Jac}\left(X_{L}^{\prime}\right)$, is isomorphic to

$$
\operatorname{Jac}\left(X_{L}^{\prime}\right) \cong \mathfrak{C}^{w}(L) / \mathfrak{C}_{-}^{w}(L)+\mathfrak{C}_{+}^{w}(L) / \Lambda / \mathfrak{C}_{-}^{w}(L)+\mathfrak{C}_{+}^{w}(L)
$$

if $L$ is good.

Suppose now that $L \in \underline{s l}_{n}\left(A_{w}\right)$. Let $\left(L\left(\lambda_{0}\right), \pi_{\lambda_{0}}\right)$ denote the basic module of the universal central extension $\widehat{S L}_{n}\left(A_{w}\right)$ of $S L_{n}\left(A_{w}\right)$. If $v_{0}$ denotes the highest weight vector of $L\left(\lambda_{0}\right)$ we define the function $\theta$ on

$$
\mathfrak{C}^{w}(L) / \mathfrak{C}_{-}^{w}(L)+\mathfrak{C}_{+}^{w}(L)
$$

in terms of representative functions as

$$
\theta(W)=e^{-\frac{1}{2} \sigma(W)}\left\langle\pi_{\lambda_{0}}(\exp W) v_{0}, v_{0}\right\rangle_{\lambda_{0}} .
$$

The term $\sigma(W)$ is defined to be $\sigma(W)=\omega\left(W_{+}, W_{-}\right)$where $\omega$ is the cocyle defining the Lie algebra extension and $W=W_{+}+W_{0}+W_{-}$is the decomposition of $W$ corresponding to $\underline{s l}_{n}(z \mathbb{C}[z])+\underline{s l}_{n}(\mathbb{C})+\underline{s l}_{n}\left(z^{-1} \mathbb{C}\left[z^{-1}\right]\right)$. Here $\langle,\rangle_{\lambda_{0}}$ denotes the contravariant, positive-definite Hermitian form on $L\left(\lambda_{0}\right)$. We show that the function $\theta$ is a theta function on the "Lie theoretic" Jacobian of $X_{L}^{\prime}$. 
Most of the material contained in this work is essentially the author's doctoral thesis given at Rutgers. He wishes to thank his thesis advisor, Nolan Wallach, for help and guidance. Discussions with Roe Goodman were also very helpful.

\section{Concepts from the Theory of Nonsingular Projective Curves.}

The purpose of this chapter is to develop notation from the theory of nonsingular projective curves.

\subsection{Nonsingular Projective Curves and Jacobian Varieties.}

The term projective curve will denote an irreducible algebraic set in $\mathbb{P}^{n}(\mathbb{C})$ of complex dimension one, with the induced topology. Most of the projective curves in this work will be given as the zero set of an irreducible homogeneous polynomial $f, f \in \mathbb{C}\left[x_{0}, x_{1}, x_{2}\right]$, in $\mathbb{P}^{2}(\mathbb{C})$.

Set

$$
X=\left\{P \in \mathbb{P}^{2}(\mathbb{C}) \mid f(P)=0\right\} .
$$

The projective curve $X$ is said to be nonsingular, if for any $P \in X$

$$
\left[\frac{\partial f}{\partial x_{0}}(P), \frac{\partial f}{\partial x_{1}}(P), \frac{\partial f}{\partial x_{2}}(P)\right] \neq 0 .
$$

However, there will be cases when the general definition (cf. Hartshorne [12], p. 31) will be necessary.

The simplicial homology groups of a nonsingular projective curve $X$ are described as follows. Since $X$ is connected, $H_{0}(X, \mathbb{Z}) \cong \mathbb{Z} . H_{1}(X, \mathbb{Z})$ is the free group on the generators $\left[a_{j}\right],\left[b_{j}\right], j=1, \ldots, g$. Thus $H_{1}(X, \mathbb{Z}) \cong \mathbb{Z}^{2 g}$. The second homology is isomorphic to the integers, $H_{2}(X, \mathbb{Z}) \cong \mathbb{Z}$, since $X$ is compact.

The integer $g$ is called the genus of the nonsingular projective curve $X$.

The generators $\left[a_{j}\right],\left[b_{j}\right]$ of $H_{1}(X, \mathbb{Z})$ can be chosen, so that the following intersection properites are satisfied (see Farkas-Kra [4], p. 54).

$$
\begin{aligned}
& a_{j} \cdot b_{k}=\delta_{j k}= \begin{cases}0, & j \neq k \\
1, & j=k\end{cases} \\
& a_{j} \cdot a_{k}=0=b_{j} \cdot b_{k} .
\end{aligned}
$$

Any basis $\left\{a_{1}, \ldots, a_{g}, b_{1}, \ldots, b_{g}\right\}$ of $H_{1}(X, \mathbb{Z})$ with these intersection properties will be called a canoncial homology basis for $X$. 
Let $\left\{\omega_{1}, \ldots, \omega_{g}\right\}$ be the unique basis for the space of holomorphic differentials with the property

$$
\int_{a_{j}} \omega_{k}=\delta_{j k}
$$

Furthermore, for this basis, the matrix $\Omega=\left(\Omega_{j k}\right)$ with

$$
\Omega_{j k}=\int_{b_{j}} \omega_{k}
$$

is symmetric with positive definite imaginary part.

The matrix $(I, \Omega)$ is called the period matrix of the holomorphic 1-forms on $X$.

Suppose $X$ is an irreducible nonsingular projective curve. Define $\Lambda=$ $\Lambda(\Omega)$ to be the lattice

$$
\mathbb{Z}^{g}+\Omega \cdot \mathbb{Z}^{g}
$$

The complex torus

$$
\operatorname{Jac}(X)=\mathbb{C}^{g} / \Lambda
$$

is called the Jacobian variety of $X$.

An equivalent definition is obtained by using sheaves (cf. Gunning [11]). Let

$$
0 \rightarrow \mathbb{Z} \rightarrow \mathfrak{o}_{X} \rightarrow \mathfrak{o}_{X}^{*} \rightarrow 0
$$

be the short exact sequence of sheaves on $X$. Here $\mathbb{Z}$ denotes the constant sheaf, $\mathfrak{o}_{X}$ the structure sheaf, and $\mathfrak{o}_{X}^{*}$ the sheaf of invertible elements of $\mathfrak{o}_{X}$ under multiplication. The map

$$
\mathfrak{o}_{X} \rightarrow \mathfrak{o}_{X}^{*}
$$

is given by the exponential map. The cohomology sequence of this short exact sequence is

$$
0 \rightarrow H^{1}(X, \mathbb{Z}) \rightarrow H^{1}\left(X, \mathfrak{o}_{X}\right) \rightarrow H^{1}\left(X, \mathfrak{o}_{X}^{*}\right) \rightarrow H^{2}(X, \mathbb{Z}) \rightarrow 0 .
$$

The sheaf theoretic Jacobian variety of $X$ is defined as

$$
\operatorname{Jac}_{\text {sheaf }}(X)=H^{1}\left(X, \mathfrak{o}_{X}\right) / H^{1}(X, \mathbb{Z})
$$

which is the kernel of the map

$$
H^{1}\left(X, o_{X}^{*}\right) \rightarrow H^{2}(X, \mathbb{Z}) .
$$




\subsection{Theta Functions.}

This section contains a definition of theta functions as given by Lang [13] and by Mumford [17].

Let $V$ be a complex vector space of dimension $n$. Suppose $\Lambda$ is a lattice in $V$, so that the factor group $V / \Lambda$ is a complex torus. A function $\Theta$ on $V$ is called a theta function with respect to $\Lambda$, if it is a quotient of entire functions, not identically zero, and

$$
\Theta(u+x)=\mathrm{e}^{2 \pi i[\ell(x, u)+J(u)]} \Theta(x), \text { all } u \in \Lambda, x \in V
$$

where $\ell$ is $\mathbb{C}$ - linear in $x$. No further assumptions are made about $\ell$ and $J$.

If $\Theta$ is a theta function with respect to $\Lambda$ then one can prove (cf. Lang $[\mathbf{1 3}]$, p. 58) that

$$
\begin{aligned}
& J(u+v)-J(u)-J(v)=\ell(u, v) \\
& \ell(u, v) \quad \equiv \ell(v, u) \quad(\bmod \mathbb{Z}) \\
& \ell(x, u+v) \quad=\ell(x, u)+\ell(x, v) \quad(\bmod \mathbb{Z}) .
\end{aligned}
$$

Then $\ell$ can be extended to a function $\ell(x, y)$ on $V \times V$, which is $\mathbb{C}$ - linear in $x$ and $\mathbb{R}$ - linear in $y . J$ can be chosen in such a way that the function

$$
K(u)=J(u)-\frac{1}{2} \ell(u, u)
$$

is $\mathbb{Z}$ - linear, and can be extended to an $\mathbb{R}$ - linear function on $V$.

In terms of $\ell$ and $K$, the relation defining a theta function can now be rewritten as

$$
\Theta(u+x)=\mathrm{e}^{2 \pi i\left[\ell(x, u)+\frac{1}{2} \ell(u, u)+K(u)\right]} \Theta(x) .
$$

\section{Affine Lie Algebras and Loop Groups.}

In this chapter we introduce some concepts from the theory of affine Lie algebras and loop groups. We use the notation of Goodman - Wallach [7] and recall some of their results.

\subsection{Affine Preliminaries.}

Let $\underline{g}$ be a simple, finite-dimensional Lie algebra over $\mathbb{C}$. Set

$$
\underline{\tilde{g}}=\underline{g} \otimes_{\mathbb{C}} \mathbb{C}\left[z, z^{-1}\right],
$$

which we consider as a subspace of the smooth maps from $S^{1}$, the circle, to $\underline{g}$. 
$\underline{\tilde{g}}$ is a Lie algebra under pointwise commutator.

Let $B$ be the Killing form on $\underline{g}$, and define a skew-symmetric bilinear form $\omega$ on $\underline{\tilde{g}}$ by

$$
\omega(X, Y)=\frac{1}{2 \pi i} \int_{0}^{2 \pi} B\left(\frac{d}{d \Theta} X\left(e^{i \Theta}\right), Y\left(e^{i \Theta}\right)\right) d \Theta
$$

for any $X, Y \in \underline{\tilde{g}}$.

Then $\omega$ is a 2 -cocycle on $\underline{\tilde{g}}$, which gives rise to a central extension of $\underline{\tilde{g}}$ denoted by $\underline{\hat{g}}$ :

$$
0 \rightarrow \mathbb{C} \rightarrow \underline{\hat{g}} \rightarrow \underline{\tilde{g}} \rightarrow 0 .
$$

As a vector space we write $\underline{\hat{g}}=\underline{\tilde{g}} \oplus \mathbb{C} c$, with commutation relations

$$
[X+a c, Y+b c]=[X, Y]_{\underline{g}}+\omega(X, Y) c,
$$

for $X, Y \in \underline{\tilde{g}}, a, b \in \mathbb{C}$. Here $[,]_{\underline{g}}$ denotes the bracket in $\underline{\tilde{g}}$, and $c$ is central.

The extended Lie algebra $\underline{\hat{g}}^{e}$ is defined to be

$$
\underline{\hat{g}^{e}}=\underline{\hat{g}} \oplus \mathbb{C} d
$$

with

$$
[d, c]=0, \quad\left[d, x \otimes z^{k}\right]=z \frac{d}{d z}\left(x \otimes z^{k}\right)=k x \otimes z^{k}, \quad x \in \underline{g}, k \in \mathbb{Z}
$$

or

$$
\left[d, X\left(e^{i \Theta}\right)\right]=\frac{1}{i} \frac{d}{d \Theta}\left(X\left(e^{i \Theta}\right)\right), \quad X \in \underline{\tilde{g}} .
$$

From the Killing form $B$ on $\underline{g}$, we obtain a bilinear form $\tilde{B}$ on $\underline{\tilde{g}}$ by integration over $S^{1}$ :

$$
\tilde{B}(X, Y)=\int_{0}^{2 \pi} B\left(X\left(e^{i \Theta}\right), Y\left(e^{i \Theta}\right)\right) d \Theta, \quad X, Y \in \underline{\tilde{g}}
$$

(Goodman - Wallach [8], p. 199). The form $\tilde{B}$ on $\underline{\tilde{g}}$ can be extended to a bilinear form $\hat{B}^{e}$ on $\underline{\hat{g}}^{e}$ by setting

$$
\begin{gathered}
\hat{B}^{e}(c, d)=1, \quad \hat{B}^{e}(c, c)=\hat{B}^{e}(d, d)=0, \quad \hat{B}^{e}(\underline{\tilde{g}}, c)=0 . \\
\hat{B}^{e}(\underline{\tilde{g}}, d)=0, \quad \hat{B}^{e}(X, Y)=\tilde{B}(X, Y), \quad X, Y \in \underline{\tilde{g}} .
\end{gathered}
$$


Fix a Cartan subalgebra $\underline{h} \subset \underline{g}$, and let $\Phi$ be the roots of $\underline{h}$ on $\underline{g}$, where $\underline{g}_{\alpha}$ denotes the $\alpha$ rootspace, $\alpha \in \Phi$. Choose $\Phi^{+}$a set of positive roots and a Chevalley basis $\left\{E_{\alpha}\right\}_{\alpha \in \Phi} \cup\left\{H_{\alpha_{j}} \mid 1 \leq j \leq \ell\right\}$ for $\underline{g}$, with $E_{\alpha} \in \underline{g}_{\alpha}$, $H_{\alpha}=\left[E_{\alpha}, E_{-\alpha}\right] \in \underline{h}$ such that $\alpha\left(H_{\alpha}\right)=2$. Here $\alpha_{1}, \ldots, \alpha_{\ell}$ denote the simple roots in $\Phi^{+}$. Put

$$
\underline{\hat{h}}^{e}=\underline{\hat{h}} \oplus \mathbb{C} c \oplus \mathbb{C} d .
$$

If $\alpha \in \underline{h}^{*}$, extend $\alpha$ to $\underline{\hat{h}}^{e}$ by setting $\alpha(c)=\alpha(d)=0$. Let $\gamma, \delta \in\left(\underline{\hat{h}}^{e}\right)^{*}$ be defined by

$$
\begin{gathered}
\gamma(\underline{h})=0, \quad \gamma(c)=1, \quad \gamma(d)=0, \\
\delta(\underline{h})=0, \quad \delta(c)=0, \quad \delta(d)=1 .
\end{gathered}
$$

Let $\tilde{\alpha}$ be the highest root in $\Phi^{+}$. Set $\alpha_{0}=\delta-\tilde{\alpha}$ and $H_{\alpha_{0}}=-H_{\tilde{\alpha}}+\frac{2 c}{(\tilde{\alpha}, \tilde{\alpha})}$. (Here $(\cdot, \cdot)$ denotes the inner product on $\underline{h}^{*}$ induced by $B$.) Denote by $\omega_{j} \in \underline{h}^{*}$, $1 \leq j \leq \ell$ the basis dual to $H_{\alpha_{j}}, 1 \leq j \leq \ell$. Define $\hat{\omega}_{j} \in\left(\underline{\hat{h}}^{e}\right)^{*}$ by the duality relations

$$
\hat{\omega}_{j}\left(H_{\alpha_{k}}\right)=\delta_{j k}, \quad 0 \leq j, k \leq \ell, \quad \hat{\omega}_{j}(d)=0 .
$$

$\lambda \in\left(\underline{\hat{h}}^{e}\right)^{*}$ is called dominant integral if $\lambda\left(H_{\alpha_{j}}\right) \in \mathbb{N}=\{0,1,2, \ldots\}$ for $j=0, \ldots, \ell$. Every such element of $\left(\underline{\hat{h}}^{e}\right)^{*}$ is of the form $n_{0} \hat{\omega}_{0}+\ldots+n_{\ell} \hat{\omega}_{\ell}+a \delta$, whre $n_{j}=\lambda\left(H_{\alpha_{j}}\right)$ and $a=\lambda(d) \in \mathbb{C}$.

Set $\underline{\hat{b}}=\underline{\hat{h}} \oplus \underline{\hat{n}}$, where $\underline{\hat{n}}$ is defined to be

$$
\underline{\hat{n}}=\sum_{\alpha \in \Phi^{+}} \underline{g}_{\alpha} \oplus \underline{g} \otimes z \mathbb{C}[z] .
$$

Given $\lambda \in\left(\underline{\hat{h}}^{e}\right)^{*}$, extend $\lambda$ to be zero on $\underline{\hat{n}}$, and form the induced $\underline{\hat{g}}^{e}$ - module

$$
M(\lambda)=U\left(\underline{\hat{g}}^{e}\right) \otimes_{U\left(\underline{\hat{b}}^{e}\right)} \mathbb{C}_{\lambda},
$$

where $\mathbb{C}_{\lambda}$ denotes the one-dimensional $\underline{\hat{b}}^{e}$ - module with action $x \rightarrow \lambda(x) \cdot 1$, and $U\left(\underline{\hat{g}}^{e}\right)$ resp. $U\left(\underline{\hat{b}}^{e}\right)$ denote the universal enveloping algebra of $\underline{\hat{g}}^{e}$ resp. $\hat{b}^{e}$. Let $L(\lambda)$ be the quotient of $M(\lambda)$ by the maximal submodule of $M(\lambda)$ which does not contain $1 \otimes 1$. When $\lambda$ is dominant integral we shall call $L(\lambda)$ a standard module with highest weight $\lambda$. by

Fix a basis $\left\{u_{j} \mid j=1, \ldots, \ell\right\}$ of $\underline{g}$ and let $\left\{u^{j} \mid j=1, \ldots, \ell\right\}$ be defined

$$
B\left(u_{j}, u^{k}\right)=\delta_{j k}
$$


Set $\rho=\frac{1}{2} \sum_{\alpha \in \Phi^{+}} \alpha$. Then the operator

$$
T=\frac{1}{2} \sum_{j=1}^{\ell} u_{j} u^{j}+\sum_{\substack{k>0 \\ k \in \mathbb{Z}}} \sum_{j=1}^{\ell}\left(u_{j} \otimes z^{-k}\right)\left(u^{j} \otimes z^{k}\right)
$$

acts on $L(\lambda)$ with action

$$
\left[\frac{1}{\lambda(c)+\frac{1}{2}}\right] T=-\alpha+\frac{\left(\lambda_{0}, \lambda_{0}+2 \rho\right)}{2 \lambda(c)+1} I,
$$

where $\lambda_{0}=\lambda_{\underline{\mid} \underline{h}}$.

\subsection{Completions of $\underline{\tilde{g}}$.}

A function $w: \mathbb{Z} \rightarrow(0, \infty)$ will be called a symmetric weight function if

$$
\begin{aligned}
& \text { (1) } w(-k)=w(k) \\
& \text { (2) } w(k+\ell) \leq w(k) w(\ell), \quad k, \ell \in \mathbb{Z} .
\end{aligned}
$$

Let $A_{w}$ be the space of functions

$$
f\left(e^{i \Theta}\right)=\sum_{k \in \mathbb{Z}} a_{k} e^{i k \Theta}
$$

on $S^{1}$ such that

$$
\|f\|_{w}:=\sum_{k \in \mathbb{Z}}\left|a_{k}\right| w(k)<\infty
$$

Then $\left(A_{w},\|\cdot\|_{w}\right)$ is a commutative Banach *-algebra under pointwise multiplication and ${ }^{*}$-operation given by complex conjugation. Since $w(k) \geq$ $w(0)^{1 / 2}, A_{w} \subset C\left(S^{1}, \mathbb{C}\right)$ with continuous inclusion.

The symmetric weight $w$ will be said to be of non-analytic type if

$$
\lim _{k \rightarrow \infty} w(k)^{1 / k}=1 \text {. }
$$

In this case the maximal ideal space of $A_{w}$ is $S^{1}$. Hence if $f \in A_{w}, f \neq 0$ on $S^{1}$, then $\frac{1}{f} \in A_{w}$ (cf. Goodman-Wallach [7], p. 128).

Let $X \in \underline{\tilde{g}}$ be given by

$$
X=\sum_{j, k} a_{j, k} u_{j} z^{k}, z=e^{i \Theta}
$$

with $\left\{u_{j}\right\}$ a basis for $\underline{g}$ and $u_{j}=u_{j}^{*}$. Define

$$
\|X\|_{w}=\sum_{j, k}\left|a_{j, k}\right| w(k) .
$$


Then $\|\cdot\|_{w}$ is a norm on $\underline{\tilde{g}}$, and we denote the completion of $\underline{\tilde{g}}$ with respect to $\|\cdot\|_{w} \underline{\tilde{g}}_{w}$.

Suppose the symmetric weight $w$ satisfies

$$
w(k) \geq C|k|^{1 / 2}, \quad k \in \mathbb{Z}
$$

for some constant $C>0$. If we define

$$
\|X+a c\|_{w}=\|X\|_{w}+|a|
$$

for $X \in \underline{\tilde{g}}, a \in \mathbb{C}$, then the completion $\underline{\hat{g}}_{w}$ of $\underline{\hat{g}}$ is a Banach Lie algebra and given by $\underline{\hat{g}}_{w}=\underline{\tilde{g}}_{w} \oplus \mathbb{C} c$ (cf. Goodman - Wallach [7] p. 83). For $\underline{g} \subset M_{n}(\mathbb{C})$ the completion $\underline{\tilde{g}}_{w}$ of $\underline{\tilde{g}}$ can also be described as

$$
\underline{\tilde{g}}_{w}=\left\{X \in M_{n}\left(A_{w}\right) \mid X(z) \in \underline{g}, z \in S^{1}\right\} .
$$

\subsection{Completion of Standard Modules.}

Suppose $\lambda$ is a dominant integral weight for $\hat{g}^{e}$, and $\left(L(\lambda), \pi_{\lambda}\right)$ is the corresponding standard $\hat{g}^{e}$-module with inner product $\langle u, v\rangle_{\lambda}$. Denote by $H^{\lambda}$ the completion of $L(\lambda)$ in the norm $\|v\|_{\lambda}=\langle v, v\rangle_{\lambda}^{1 / 2}$. Let $A$ be the closure of

$$
I+\left(\frac{1}{\lambda(c)+\frac{1}{2}}\right) T
$$

in $H_{\lambda}$. Define the powers $A^{r}, r \in \mathbb{R}$, by the spectral theorem, and set

$$
\|v\|_{\sigma, t}=\left\|\exp \left(t A^{1 / \sigma}\right) v\right\|_{\lambda}
$$

for $v \in L(\lambda), t \geq 1$ and $\sigma \geq 1$. Denote by $S_{\sigma, t}^{\lambda}$ the completion of $L(\lambda)$ relative to this norm. Then

$$
S_{\sigma}^{\lambda}=\bigcap_{t>0} S_{\sigma, t}^{\lambda}
$$

will be called the space of Gevrey vectors of order $\sigma$ for $A$.

2.4. The Group $\hat{G}_{w}$.

Let $f_{1}, \ldots, f_{\alpha}$ be polynomials on $M_{n}(\mathbb{C})$. Assume that

$$
G=\left\{g \in S L_{n}(\mathbb{C}) \mid f_{j}(g)=0, j=1, \ldots, d\right\}
$$

is a connected and simply connected group with simple Lie algebra $\underline{g}$. Also assume that if $g \in G$, then the conjugate-transpose $g^{*} \in G$. Set

$$
\widetilde{S L}_{n}=S L_{n}\left(A_{w}\right)=\left\{g \in M_{n}\left(A_{w}\right) \mid g^{-1} \in M_{n}\left(A_{w}\right), \operatorname{det}(g)=1\right\}
$$


for $w$ a weight of non-analytic type. Then $\tilde{G}_{w}$, with

$$
\tilde{G}_{w}=\left\{g \in S L_{n}\left(A_{w}\right) \mid f_{j}(g(z))=0, j=1, \ldots, d, z \in S^{1}\right\},
$$

is a closed Banach Lie subgroup of $S L_{n}\left(A_{w}\right)$ with Lie algebra

$$
\underline{\tilde{g}}_{w}=\left\{X \in M_{n}\left(A_{w}\right) \mid \mathrm{d} f_{j}(X(z))=0, j=1, \ldots, d, z \in S^{1}\right\} .
$$

If $\lambda_{0}, \lambda_{1}, \ldots, \lambda_{\ell}$ are the fundamental weights of $\underline{\hat{g}}$, set

$$
S_{\sigma}=\bigoplus_{j=0}^{\ell} S_{\sigma}^{\lambda_{j}}, \quad \pi=\underset{j=0}{\oplus} \pi_{\lambda_{j}}
$$

Let $\hat{G}_{w}$ be the group generated by

$$
\left\{\exp (\pi(X)) \mid X \in \underline{\hat{g}}_{w}\right\} .
$$

Let $Z$ denote the center of $\hat{G}_{w}$. Then $Z$ is a closed Lie subgroup of $\hat{G}_{w}$ with Lie algebra $\mathbb{C}$. The identity component of $Z$ is

$$
Z^{0}=\{\exp (t \pi(c)) \mid t \in \mathbb{C}\}
$$

Assume there exists a $\sigma, 1<\sigma<2$, such that the weight $w$ satisfies the inequality

$$
C_{t} \exp \left(t|k|^{1 / \sigma}\right) \leq w(k) \leq M_{\epsilon} \exp (\epsilon|k|)
$$

for all $t>0, \epsilon>0$ where $C_{t}, M_{\epsilon}$ are positive constants. Then we have an exact sequence of Lie group homomorphisms

$$
1 \rightarrow Z^{0} \rightarrow \hat{G}_{w} \stackrel{p_{\zeta}}{\rightarrow} \tilde{G}_{w} \rightarrow 1
$$

whose differentials give the Lie algebra exact sequence

$$
0 \rightarrow \mathbb{C} c \rightarrow \underline{\hat{g}}_{w} \stackrel{\mathrm{p}}{\rightarrow}_{\underline{\tilde{g}}_{w}} \rightarrow 0
$$

(cf. Goodman-Wallach [7], p. 111).

\section{Isospectral Curves and Their Jacobians.}

\subsection{Isospectral Curves.}

Throughout this chapter we make the following assumptions:

3.1.1. There exists $\sigma, 1<\sigma<2$, such that the symmetric weight $w$ satisfies the inequalities

$$
C_{t} \exp \left(t|k|^{1 / \sigma}\right) \leq w(k) \leq M_{\epsilon} \exp (\epsilon|k|)
$$


for all $t, \epsilon>0$ with constants $C_{t}, M_{\epsilon}>0$.

Suppose $L \in \underline{\tilde{g}}, \underline{\tilde{g}}=M_{n}(\mathbb{C}) \otimes_{\mathbb{C}} \mathbb{C}\left[z, z^{-1}\right]$, is given by

$$
L=L(z)=\sum_{j=-p}^{q} \ell_{j} z^{j}, \ell_{j} \in M_{n}(\mathbb{C}), j=-p, \ldots, q, p+q \geq 1 .
$$

3.1.2. The characteristic polynomials of $L_{0}(z)=z^{p} L(z)$ and $L_{\infty}\left(z^{-1}\right)=$ $z^{-q} L(z), f_{0}(z, \lambda)=\operatorname{det}\left(L_{0}(z)-\lambda\right)$ and $f_{\infty}\left(z^{-1}, \lambda\right)=\operatorname{det}\left(L_{\infty}\left(z^{-1}\right)-\lambda\right)$ are irreducible.

Define the quasi-affine curve $X^{a}$ to be

$$
X^{a}=\left\{(z, \lambda) \in \mathbb{C}^{*} \times \mathbb{C} \mid f(z, \lambda)=\operatorname{det}(L(z)-\lambda)=0\right\}
$$

Then $X^{a}$ is irreducible. Let $X_{L}$ denote the corresponding projective curve of $X^{a} . X_{L}$ is obtained by homogenizing

$$
L_{0}(z)-\lambda=\sum_{j=0}^{p+q} \ell_{j-p} z^{j}-\lambda
$$

and setting

$$
\begin{gathered}
M\left(x_{0}, x_{1}, x_{2}\right)=\sum_{j=0}^{p+q} \ell_{j-p} z^{j} t^{p+q-j}+t^{p+q-1} \lambda, \\
x_{0}=z, x_{1}=\lambda, x_{2}=t .
\end{gathered}
$$

The isospectral curve $X_{L}$ is defined to be

$$
X_{L}=\left\{\left(x_{0}, x_{1}, x_{2}\right) \mid \operatorname{det} M\left(x_{0}, x_{1}, x_{2}\right)=0\right\} \subset \mathbb{P}^{2}(\mathbb{C}) .
$$

Set

$$
\begin{gathered}
X_{0}=\left\{(z, \lambda) \in \mathbb{C} \times \mathbb{C} \mid f_{0}(z, \lambda)=0\right\}, \\
X_{\infty}=\left\{\left(z^{-1}, \lambda\right) \in \mathbb{C} \times \mathbb{C} \mid f_{\infty}\left(z^{-1}, \lambda\right)=0\right\} .
\end{gathered}
$$

Then $X^{a} \subset X_{L}$ and we have the following maps

$$
\psi_{0}: X^{a} \rightarrow X_{0},(z, \lambda) \rightarrow\left(z, z^{p} \lambda\right)
$$




$$
\psi_{\infty}: X^{a} \rightarrow X_{\infty},\left(z^{-1}, \lambda\right) \rightarrow\left(z^{-1}, z^{-q} \lambda\right)
$$

We also assume

3.1.3. $X_{0} \cap X_{\infty}$ is nonsingular or equivalently $d f(z, \lambda)_{\mid X_{0} \cap X_{\infty}} \neq 0$.

If $f(z, \lambda)$ has singularities on $X_{L}$, denote by $S$ the set of singular points of $X_{L}$.

Theorem 3.1.4. Let $L$ be as above. Then there exists a nonsingular model $X_{L}^{\prime}$ of $X_{L}$ and a projection map $p: X_{L}^{\prime} \rightarrow X_{L}$. Set $S^{\prime}=p^{-1}(S)$, then $p: X_{L}^{\prime}-S^{\prime} \rightarrow X_{L}-S$ is biholomorphic.

Proof. (Cf. Serre [24], pp. 67/68.)

In this chapter we will be analyzing two classes of curves:

3.1.5. $X_{L}$ is nonsingular, i.e. $S=0$. Then $X_{L}^{\prime}=X_{L}$.

3.1.6. $L$ is given by

$$
\begin{aligned}
L(z) & =\ell_{0}+\ell_{1} z+\ell_{2} z^{2}, \\
\ell_{0} & =E_{1, n}, \\
\ell_{1} & =\sum_{j=1}^{n-1}\left(a_{j} E_{j, j+1}+b_{j} E_{j+1, j}\right)+\sum_{j=1}^{n} d_{j} E_{j, j}, \\
\ell_{2} & =E_{n, 1} \\
\prod_{j=1}^{n-1} a_{j} & \neq 0, \quad \prod_{j=1}^{n-1} b_{j} \neq 0 .
\end{aligned}
$$

In Section 3.3 we will give a condition in terms of the $a_{j}$ 's, $b_{j}$ 's and $d_{j}$ 's which guarantees the nonsingularity of $X_{0} \cap X_{\infty}, X_{0} \cap X_{\infty} \subset X_{L}$.

We associate to $X_{L}$ a line bundle $E_{L} \rightarrow X_{L}$ as follows: For $x \in X_{L}$ define

$$
E_{L}(x)=\operatorname{Ker}(L(z(x))-\lambda(x))
$$

Then we have

Theorem 3.1.7. If $f(z, \lambda)$ is nonsingular on $X_{L}$, then $E_{L}(x)$ is one-dimensional for any $x \in X_{L}$ and the map

$$
x \rightarrow E_{L}(x)
$$

is holomorphic from $X_{L}$ to $\mathbb{P}^{n}(\mathbb{C})$.

Proof. (a) Suppose $z_{0} \in \mathbb{C}$ is such that $L\left(z_{0}\right)$ does not have simple spectrum. If $\lambda_{0}$ is a multiple eigenvalue of $L\left(z_{0}\right)$, then by the nonsingularity of $f(z, \lambda)$ 
we obtain

$$
\frac{\partial f}{\partial z}(z, \lambda)_{\mid\left(z_{0}, \lambda_{0}\right)} \neq 0, \quad \frac{\partial f}{\partial \lambda}(z, \lambda)_{\mid\left(z_{0}, \lambda_{0}\right)}=0 .
$$

Define $\mathbb{C}_{\lambda_{0}}^{n}$ to be the generalized eigenspace of $\lambda_{0}$

$$
\mathbb{C}_{\lambda_{0}}^{n}=\left\{v \in \mathbb{C}^{n} \mid\left(L\left(z_{0}\right)-\lambda_{0}\right)_{\nu}^{r}=0, \quad \text { for some } r \in \mathbb{N}\right\} .
$$

Assume there exist linearly independent vectors $v_{1}, v_{2} \in \mathbb{C}_{\lambda_{0}}^{n}$ such that $L\left(z_{0}\right) v_{j}=\lambda_{0} v_{j}, \quad j=1,2$. Extend $v_{1}, v_{2}$ to a basis $\left\{v_{j}\right\}$ of $\mathbb{C}^{n}$. Then

$$
\begin{gathered}
\operatorname{det}\left(L\left(z_{0}\right)-\lambda\right)=\left(\lambda-\lambda_{0}\right)^{2} \Phi(\lambda) \\
\left(L(z)-\lambda_{0}\right) v_{1} \wedge \ldots \wedge\left(L(z)-\lambda_{0}\right) v_{n}=f\left(z, \lambda_{0}\right) v_{1} \wedge \ldots \wedge v_{n},
\end{gathered}
$$

which yields $f\left(z, \lambda_{0}\right)=\left(z-z_{0}\right)^{2} g(z)$, contradicting $\frac{\partial f}{\partial z}(z, \lambda)_{\mid\left(z_{0}, \lambda_{0}\right)} \neq 0$.

(b) Denote by $\mathfrak{A} \mathfrak{d}\left(M\left(x_{0}, x_{1}, x_{2}\right)\right)$ the classical adjoint (cofactor matrix) of $M\left(x_{0}, x_{1}, x_{2}\right)$ as in the definition of $X_{L}$ and let $e_{j}(x)=e_{j}\left(x_{0}, x_{1}, x_{2}\right)$ be the $\mathrm{j}$-th column of $\mathfrak{A d}\left(M\left(x_{0}, x_{1}, x_{2}\right)\right)$. If $\operatorname{det} M\left(x_{0}, x_{1}, x_{2}\right)=0$ then by (a) $\operatorname{dim} \operatorname{Ker} M\left(x_{0}, x_{1}, x_{2}\right)=1$ hence $\operatorname{rank} M\left(x_{0}, x_{1}, x_{2}\right)=n-1$. Consequently there exists $j, 1 \leq j \leq n$, such that $e_{j}(x) \neq 0$. Set

$$
U_{j}=\left\{x \in X_{L} \mid e_{j}(x) \neq 0\right\}
$$

Then $\bigcup_{j=1}^{n} U_{j}=X_{L}$ and the map

$$
X_{L} \rightarrow \mathbb{P}^{n}(\mathbb{C}), \quad x \rightarrow e_{j}(x)
$$

is polynomial and homogeneous. On $U_{j} \cap U_{k}$ we have $e_{j}(x)=g_{j, k} e_{k}(x)$ with $g_{j, k}$ holomorphic.

Theorem 3.1.8. Let $L$ be as in 3.1.6. If $x \in X_{0} \cap X_{\infty}$, then $E_{L}(x)$ is one-dimensional and the map

$$
x \rightarrow E_{L}(x)
$$

is rational from $X_{L}$ to $\mathbb{P}^{n}(\mathbb{C})$.

Proof. From 3.1.7 (a) it follows that $E_{L}(x)$ is one-dimensional for $x \in X_{0} \cap$ $X_{\infty}$, since $X_{0} \cap X_{\infty}$ is nonsingular. Define $\Phi$ on the Zariski-open set $X_{0} \cap X_{\infty}$ as

$$
\Phi: X_{0} \cap X_{\infty} \rightarrow \mathbb{P}^{n}(\mathbb{C}), x \rightarrow e_{j}(x) \quad(\text { see } 3.1 .7(\mathrm{~b}))
$$


If $A \subset \Phi\left(X_{0} \cap X_{\infty}\right) \cap \mathbb{P}^{n}(\mathbb{C})$ is closed, then $\Phi^{-1}(A)$ is closed, since $\Phi$ is polynomial and homogeneous. Thus $\Phi$ is continuous in the Zariski-topology. Suppose $V \subset \mathbb{P}^{n}(\mathbb{C})$ is open and $r: V \rightarrow \mathbb{C}$ is a regular function on $V$, i.e. for $P \in U \subset V, U$ open, there existhomogeneou s polynomials $g, h$ of the same degree, such that $h$ is nowhere zero on $U$, and $r=\frac{g}{h}$ on $U$. Consequently $r \circ \Phi: \Phi^{-1}(V) \rightarrow \mathbb{C}$ is regular. If $x \in \Phi^{-1}(U)$, then there exists $j, 1 \leq j \leq n$, so that $x \in U_{j}$ (3.1.7 (b)). Thus $r \circ \Phi=\frac{g \circ \Phi}{h \circ \Phi}$ and $g \circ \Phi, h \circ \Phi$ are homogeneous polynomials of the same degree.

Moreover, $h \circ \Phi$ is nowhere zero on $U_{j} \cap \Phi^{-1}(V)$. Hence $\Phi$ is a rational $\operatorname{map} \Phi: X_{L} \rightarrow \mathbb{P}^{n}(\mathbb{C})$ (cf. Hartshorne [12], pp. 14-24).

Theorem 3.1.9. Suppose $L$ is as in 3.1.6. Let $\Phi: X_{L} \rightarrow \mathbb{P}^{n}(\mathbb{C}), x \rightarrow$ $E_{L}(x)$ be the rational map in 3.1.8. Then the induced rational map $\Phi^{\prime}$ : $X_{L}^{\prime} \rightarrow \mathbb{P}^{n}(\mathbb{C})$ is everywhere regular.

Proof. (Cf. Mumford [16], pp. 166/167.)

\subsection{The Algebraic Curve $X_{L}$ (Nonsingular Case).}

Let $L \in \underline{\tilde{g}}$ be given by

$$
L=L(z)=\sum_{j=-p}^{q} \ell_{j} z^{j}, \ell_{j} \in M_{n}(\mathbb{C}), j=-p, \ldots, q, p+q \geq 1 .
$$

Theorem 3.2.1. Let $L$ be as above. Suppose $f(z, \lambda)$ is nonsingular on $X_{L}$. Then the genus of $X_{L}, g\left(X_{L}\right)$, is equal to

$$
g\left(X_{L}\right)=\frac{1}{2} n(n-1)(p+q)-n+1 .
$$

Proof. (a) We may replace $L(z)$ by $L_{0}(z)$. Let $p_{1}: X_{L} \rightarrow \mathbb{P}^{1}$ be the projection map corresponding to $z$, so that $p_{1}(z, \lambda)=z$. Then $\operatorname{deg} p_{1}=1$. By Hurwitz formula (cf. Farkas - Kra [4] p. 18) we have

$$
2 g\left(X_{L}\right)-2=n(2 \gamma-2)+B=-2 n+B,
$$

since the genus of $\mathbb{P}^{1}, \gamma\left(\mathbb{P}^{1}\right)=0$. To establish the result we observe that the total branch number, $B$, is given by

$$
B=\sum_{z \in \mathbb{C}} \sum_{\lambda \in \operatorname{Spec} L(z)}\{\operatorname{mult}(\lambda)-1\}
$$


where $\operatorname{Spec} L(z)$ denotes the spectrum of $L(z)$ and $\operatorname{mult}(\lambda)$ the multiplicity of $\lambda$. We now use this observation to show that

$$
B=n(n-1)(p+q) .
$$

(b) Suppose $A \in M_{n}(\mathbb{C})$. Define $Q(A)$ by $\operatorname{det}(\operatorname{ad} A-t I)=t^{n} Q(A)+$ higher order terms in $t$.

Then $Q(A)$ is polynomial and if $A$ has eigenvalues $\lambda_{1}, \ldots, \lambda_{n}$ counting multiplicities, then

$$
Q(A)=\prod_{j \neq k}\left(\lambda_{j}-\lambda_{k}\right)
$$

Thus $Q(t A)=t^{n(n-1)} Q(A)$. Since there exists $z_{0} \in \mathbb{C}$ such that $L\left(z_{0}\right)$ has simple spectrum we have $Q(L(z)) \not \equiv 0$ and

$$
\begin{aligned}
Q(L(z)) & =Q\left(z^{p+q} \ell_{q}+\text { lower order terms in } z\right) \\
& =z^{n(n-1)(p+q)} Q\left(\ell_{q}\right)+\text { lower order terms in } z .
\end{aligned}
$$

If $\ell_{q}$ does not have simple spectrum, then if $L\left(z_{0}\right)$ does replace $L(z)$ by $L\left(z+z_{0}\right)$ and consider $L_{\infty}\left(\left(z+z_{0}\right)^{-1}\right)$ to obtain

$$
\operatorname{deg} Q(L(z))=n(n-1)(p+q) .
$$

(c) Assume $L\left(z_{0}\right)$ has a multiple eigenvalue for some $z_{0} \in \mathbb{C}$. We assert that

$$
\operatorname{ord}_{z_{0}} Q(L(z))=\sum_{\lambda \in \operatorname{Spec} L\left(z_{0}\right)}\{\operatorname{mult}(\lambda)-1\}
$$

We may assume $z_{0}=0$, otherwise we can replace $L(z)$ by $L\left(z+z_{0}\right)$. Let $\lambda_{1}, \ldots, \lambda_{n}$ be the eigenvalues of $L(0)$ counting multiplicities. If $\lambda \in \operatorname{Spec} L(0)$ and $r=\operatorname{mult}(\lambda), r>1$, we have

$$
f(0, \lambda)=\prod\left(\lambda_{i}-\lambda\right), \frac{\partial f}{\partial \lambda}(0, \lambda)=0, \frac{\partial f}{\partial z}(0, \lambda) \neq 0 .
$$

We may also assume $\lambda=0$, otherwise we can shift by $-\lambda$. Then by the Inverse Function Theorem $f(z, \lambda)$ defines $z=z(\lambda)$ in some disc in the $\lambda$ parameter with $z(0)=0$. We assert that $\operatorname{ord}_{0} z(\lambda)=r$. We have

$$
\frac{\partial^{s}}{\partial \lambda^{s}} f(z, \lambda)_{\left.\right|_{(0,0)}}=0, s<r, \frac{\partial^{r}}{\partial \lambda^{r}} f(z, \lambda)_{\left.\right|_{(0,0)}} \neq 0 \text {. }
$$

Then

$$
0=\frac{\mathrm{d}}{\mathrm{d} \lambda} f(z, \lambda)_{\left.\right|_{\lambda=0}}=\frac{\partial}{\partial z} f(z(\lambda), \lambda) z^{\prime}(\lambda)_{\left.\right|_{\lambda=0}}+\frac{\partial}{\partial \lambda} f(z(\lambda), \lambda)_{\left.\right|_{\lambda=0}}
$$


giving $z^{\prime}(0)=0$ by equation (1), and more generally

$$
0=\frac{\mathrm{d}^{s}}{\mathrm{~d} \lambda^{s}} f(z, \lambda)_{\left.\right|_{\lambda=0}}=\frac{\partial}{\partial z} f(z(\lambda), \lambda) z^{(s)}(\lambda)_{\left.\right|_{\lambda=0}}+\frac{\partial^{s}}{\partial \lambda^{s}} f(z(\lambda), \lambda)_{\left.\right|_{\lambda=0}}
$$

yielding $z^{(s)}(0)=0$ for $s<r$. Thus

$$
z(\lambda)=a_{r} \lambda^{r}+\text { higher order terms in } \lambda,
$$

which gives $\lambda=\lambda\left(z^{1 / r}\right), \lambda(0)=0, \lambda^{\prime}(0) \neq 0$, with $\lambda$ holomorphic in a neighborhood of zero. If $\lambda_{1}, \ldots, \lambda_{k}$ are the distinct eigenvalues of $L(0)$ with multiplicities $r_{1}, \ldots, r_{k}$ respectively, then $\lambda_{j}=\lambda_{j}\left(z^{1 / r_{j}}\right), 1 \leq j \leq k$, with $\lambda_{j}$ holomorphic near 0 . This implies

$$
Q(L(z))=\prod_{j=1}^{k} z^{r_{j}\left(r_{j}-1\right) / r_{j}} g(z), \quad g(0) \neq 0
$$

with $g(z)$ polynomial, since $Q(L(z))$ is polynomial. Thus

$$
\operatorname{ord}_{0} Q(L(z))=\sum_{j=1}^{k}\left(r_{j}-1\right)
$$

Hence

$$
\sum_{z_{0} \in \mathbb{C}} \operatorname{ord}_{z_{0}} Q(L(z))=\operatorname{deg} Q(L(z))=n(n-1)(p+q)
$$

\subsection{The Isospectral Curve $X_{L}$ (Tridiagonal Case).}

Let $L(z)=\ell_{0}+\ell_{1} z+\ell_{2} z^{2}$ be tridiagonal as in 3.1. Then

$$
z^{-1} L(z)-\lambda=\left[\begin{array}{cccccc}
d_{1}-\lambda & a_{1} & \ldots & & z^{-1} \\
b_{1} & d_{2}-\lambda & a_{2} & \ldots & \vdots \\
0 & b_{2} & d_{3}-\lambda & \cdots & \\
\vdots & & & & a_{n-1} \\
z & \cdots & & b_{n-1} & d_{n}-\lambda
\end{array}\right]
$$

The determinant of $z^{-1} L(z)-\lambda$ is given by

$$
f(z, \lambda)=(-1)^{n+1}\left[\left(\prod_{j=1}^{n-1} a_{j}\right) z+\left(\prod_{j=1}^{n-1} b_{j}\right) z^{-1}-P(\lambda)\right]
$$


where $P(\lambda)$ is a polynomial of degree $n$ with leading coefficient 1 . Set $A=$ $\prod_{n=1}^{n-1} a_{j}, B=\prod_{j=1}^{n-1} b_{j}$. Then by assumption $A \neq 0, B \neq 0$ and the curve $X_{L}$ is defined by

$$
A z+B z^{-1}-P(\lambda)=0
$$

Equivalently,

$$
z(\lambda)=\frac{1}{2 A}\left[P(\lambda) \pm \sqrt{P(\lambda)^{2}-4 A B}\right]=\frac{1}{\frac{1}{2 A}\left[P(\lambda) \mp \sqrt{P(\lambda)^{2}-4 A B}\right]} .
$$

Theorem 3.3.1. Let $L$ be as above and suppose $X_{L}$ is nonsingular on $X_{0} \cap$ $X_{\infty}$. Then the curve $X_{L}$ is hyperelliptic of genus $g\left(X_{L}^{\prime}\right)=n-1$.

Proof. (Van Moerbeke-Mumford [14], pp. 125/126.)

The assumption that $X_{L}$ is nonsingular on $X_{0} \cap X_{\infty}$ was added and is necessary as the following example shows.

Consider the matrix:

$$
L(z)-\lambda=\left[\begin{array}{ccc}
-\lambda & 1 & z^{-1} \\
1 & -\lambda & 1 \\
z & 1 & -\lambda
\end{array}\right]
$$

Then $f(z, \lambda)=-\lambda^{3}+3 \lambda+z+z^{-1}$ and consequently $f(z, \lambda)$ is irreducible. We have

$$
\frac{\partial f}{\partial z}(z, \lambda)=1-z^{-2}, \frac{\partial f}{\partial \lambda}(z, \lambda)=-3 \lambda^{2}+3,
$$

which implies that $(z, \lambda)=(-1,1)$ and $(z, \lambda)=(1,-1)$ are singular points of $X_{L}$. Moreover, $A=1=B$ and

$$
P(\lambda)^{2}-4=(\lambda-1)^{2}(\lambda+2)(\lambda+1)^{2}(\lambda-2), P^{\prime}(\lambda)=3\left(\lambda^{2}-1\right) .
$$

Thus $P(\lambda)^{2}-4$ and $P^{\prime}(\lambda)$ have a non-constant factor in common.

To allow singularities only on $X_{L} \backslash X_{0} \cap X_{\infty}$, i.e. at $z=0$ or $z^{-1}=0$, the condition we need is as follows:

Set

$$
f(z, \lambda)=A z+B z^{-1}-P(\lambda)
$$

Then

$$
\frac{\partial f}{\partial z}(z, \lambda)=A-B z^{-2}, \frac{\partial f}{\partial \lambda}(z, \lambda)=-P^{\prime}(\lambda)
$$


If $\frac{\partial f}{\partial z}=0$, then $z= \pm \sqrt{\frac{A}{B}}$. Hence the condition we obtain is, that the polynomials

$$
P(\lambda) \pm 2 \sqrt{A B}, P^{\prime}(\lambda)
$$

have no non-constant factor in common. If

$$
R=R\left(P(\lambda)^{2}-4 A B, P^{\prime}(\lambda)\right)
$$

denotes the resultant of $P(\lambda)^{2}-4 A B$ and $P^{\prime}(\lambda)$, then the above condition is equivalent to $R \neq 0$. The resultant is a polynomial in the

$$
a_{1}, \ldots, a_{n-1}, b_{1}, \ldots, b_{n-1}, d_{1}, \ldots, d_{n}
$$

(cf. van der Waerden [25], p. 108). The zeroset of $R$

$$
Z(R)=\left\{\left(a_{1}, \ldots, a_{n-1}, b_{1}, \ldots, b_{n-1}, d_{1}, \ldots, d_{n}\right) \in \mathbb{C}^{3 n-2} \mid R=0\right\}
$$

is an affine algebraic set of $\mathbb{C}^{3 n-2}$ of codimension 1 , the zeroset

$$
Z(A, B)=\left\{\left(a_{1}, \ldots, a_{n-1}, b_{1}, \ldots, b_{n-1}, d_{1}, \ldots, d_{n}\right) \in \mathbb{C}^{3 n-2} \mid A B=0\right\}
$$

has codimension 1 in $\mathbb{C}^{3 n-2}$. Therefore for $\left(a_{1}, \ldots, a_{n-1}, b_{1}, \ldots, b_{n-1}, d_{1}, \ldots, d_{n}\right)$ in the complement of $Z(R) \cup Z(A, B)$ we have the nonsingularity on $X_{0} \cap X_{\infty}$.

\subsection{Lie Theoretic Construction of the Jacobian Variety.}

Let $L$ satisfy 3.1 .2 and 3.1.3. Denote by $\underline{\tilde{g}}_{w,+}$ resp. $\underline{\tilde{g}}_{w,-}$ the completions of $M_{n}(\mathbb{C}[z])$ resp. $M_{n}\left(\mathbb{C}\left[z^{-1}\right]\right)$ with respect to the weight $w$. Let $\mathfrak{C}^{w}(L)$ denote the centralizer of $L$ in $\underline{\tilde{g}}_{w}$. The centralizers of $L$ in $\underline{\tilde{g}}_{w,+}$ resp. $\underline{\tilde{g}}_{w,-}$ will be denoted $\mathfrak{C}_{+}^{w}(L)$ resp. $\mathfrak{C}_{-}^{w}(L)$.

Theorem 3.4.1. Let $L$ be as above. Suppose $f(z, \lambda)$ is nonsingular on $X_{L}$ for all $z \in S^{1}$. If $M \in \mathfrak{C}^{w}(L)$, then

$$
M(z)=\sum_{k=1}^{n-1} c_{k}(z) L^{k}(z)
$$

with $c_{k}(z) \in A_{w}$.

Proof. (a) Suppose $\left(z_{0}, \lambda_{0}\right) \in X_{L}$ and $\mathrm{d} f_{\mid\left(z_{0}, \lambda_{0}\right)} \neq 0$. Then $I, L\left(z_{0}\right), \ldots, L^{n-1}\left(z_{0}\right)$ is a basis of the centralizer of $L\left(z_{0}\right)$ in $M_{n}(\mathbb{C})$.

Suppose $L\left(z_{0}\right)$ is in Jordan canonical form. By 3.1.7 (a) we have $\operatorname{dim} \operatorname{Ker}\left(L\left(z_{0}\right)-\lambda_{0}\right)=1$. Thus there exists exactly one Jordan block for the 
eigenvalue $\lambda_{0}$ of $L\left(z_{0}\right)$. If $\lambda_{1}, \ldots, \lambda_{k}, 1 \leq k \leq n$, are the distinct eigenvalues of $L\left(z_{0}\right)$ with multiplicities $r_{1}, \ldots, r_{k}$ respectively, then (notation as in 3.1.7 (a))

$$
\mathbb{C}^{n}=\bigoplus_{j=1}^{k} \mathbb{C}_{\lambda_{j}}^{n}, \operatorname{dim}\left(\mathbb{C}_{\lambda_{j}}^{n}\right)=r_{j}, 1 \leq j \leq k .
$$

We note that if $A \in M_{n}(\mathbb{C})$ commutes with $L\left(z_{0}\right)$, then $A \mathbb{C}_{\lambda_{j}}^{n} \subset \mathbb{C}_{\lambda_{j}}^{n}$ for $1 \leq j \leq k$. Hence it is enough to consider the dimension of the centralizer of $L\left(z_{0}\right)$ on $\mathbb{C}_{\lambda^{\prime}}^{n}$ for $1 \leq j \leq k$. Denote by $L\left(z_{0}\right)_{\left.\right|_{\mathbb{C}_{j}^{n}} ^{n}}$ the restriction of $L\left(z_{0}\right)$ to $\mathbb{C}_{\lambda_{j}}^{n}$. Since $\mathbb{C}_{\lambda_{j}}^{n}$ is a cyclic $\mathbb{C}\left[L\left(z_{0}\right)_{\mathbb{|}_{\lambda_{\lambda_{j}}^{n}}^{n}}\right]$-module the centralizer of $L\left(z_{0}\right)_{\mathbb{I}_{\mathbb{C}_{j}^{n}}^{n}}$ is isomorphic to

$$
\mathbb{C}\left[L\left(z_{0}\right)_{\left.\right|_{\mathbb{C}_{\lambda_{j}}^{n}}}\right] /\left(m_{j}\right)
$$

where $m_{j}$ denotes the minimal polynomial of $L\left(z_{0}\right)_{\left.\right|_{\mathbb{C}_{\lambda_{j}}^{n}}}$. Since $\operatorname{deg} m_{j}=r_{j}$ it follows

$$
\operatorname{dim} \mathbb{C}\left[L\left(z_{0}\right)_{\left.\right|_{\mathbb{C}_{j}^{n}}}\right] /\left(m_{j}\right)=r_{j}, \quad 1 \leq j \leq k
$$

Thus the dimension of the centralizer of $L\left(z_{0}\right)$ in $M_{n}(\mathbb{C})$ equals $\sum_{j=1}^{n} r_{j}=n$. The minimal polynomial of $L\left(z_{0}\right)$ on $\mathbb{C}^{n}, m\left(L\left(z_{0}\right)\right)$, is $m\left(L\left(z_{0}\right)\right)=\prod_{j=1}^{k} m_{j}$. Hence $\operatorname{deg}\left(m\left(L\left(z_{0}\right)\right)\right)=n$ which implies $I, L\left(z_{0}\right), \ldots, L^{n-1}\left(z_{0}\right)$ are linearly independent.

(b) Suppose $M(z) \in \mathfrak{C}^{w}(L)$. Then, if $f(z, \lambda)=0, \mathrm{~d} f(z, \lambda) \neq 0$, we obtain $M(z) \wedge\left(I \wedge L(z) \wedge \ldots \wedge L^{n-1}(z)\right) \equiv 0$ in $\Lambda^{n+1} M_{n}(\mathbb{C})$ for $z \in S^{1}$. Hence

$$
M(z)=\sum_{k=0}^{n-1} c_{k}(z) L^{k}(z), \quad z \in S^{1} .
$$

(c) From the equation (2) one obtains

$c_{k}(z)\left(I \wedge\left(L(z) \wedge \ldots \wedge L^{n-1}(z)\right)\right)=(-1)^{k}\left(M(z) \wedge I \wedge \ldots \wedge \hat{L^{k}}(z) \wedge \ldots \wedge L^{n-1}(z)\right)$.

The map

$$
z \rightarrow M(z) \wedge I \wedge \ldots \wedge \hat{L}^{k}(z) \wedge \ldots \wedge L^{n-1}(z)
$$

from $S^{1} \rightarrow \Lambda^{n} M_{n}(\mathbb{C})$ has coordinates in $A_{w}$; the map

$$
z \rightarrow I \wedge L(z) \wedge \ldots \wedge L^{n-1}(z)
$$


from $S^{1} \rightarrow \Lambda^{n} M_{n}(\mathbb{C})$ has polynomial coordinates and is nowhere 0 . Set $X_{J}=X_{j_{1}} \wedge \ldots \wedge X_{j_{n}}$, with $X_{1}, \ldots, X_{n^{2}}$ a basis of $M_{n}(\mathbb{C})$. Then

$$
I \wedge L(z) \wedge \ldots \wedge L^{n-1}(z)=\sum b_{J}(z) X_{J}, b_{J}(z) \in \mathbb{C}\left[z, z^{-1}\right]
$$

$$
\overline{I \wedge L(z) \wedge \ldots \wedge L^{n-1}(z)} \cdot c_{k}(z)\left(I \wedge L(z) \wedge \ldots \wedge L^{n-1}(z)\right)=\sum \overline{b_{J}(z)} b_{J}(z) c_{k}(z)
$$

and

$$
\begin{aligned}
\overline{I \wedge L(z) \wedge \ldots \wedge L^{n-1}(z)} \cdot(-1)^{k}\left(M(z) \wedge I \wedge \ldots \wedge \hat{L^{k}}(z) \wedge \ldots \wedge L^{n-1}(z)\right)= \\
=\sum \overline{b_{J}(z)} \Phi_{J}(z)
\end{aligned}
$$

with $\Phi_{J}(z) \in A_{w}$. Now $\sum \overline{b_{J}(z)} b_{J}(z)=\sum\left|b_{J}(z)\right|^{2} \neq 0, z \in S^{1}$. Hence

$$
c_{k}(z)=\frac{\sum \overline{b_{J}(z)} \Phi_{J}(z)}{\sum\left|b_{J}(z)\right|^{2}} \in A_{w} .
$$

From the proof of the theorem, it follows $\mathfrak{C}^{w}(L)$ and $\mathfrak{C}_{ \pm}^{w}(L)=\mathfrak{C}^{w}(L) \cap$ $\underline{\tilde{g}}_{w, \pm}$ are abelian Lie algebras.

Lemma 3.4.2. Let $L$ be as above. If $A \in M_{n}(\mathbb{C})$ is such that $[A, L]_{\underline{\tilde{g}}}=0$, then $A=a \cdot I, a \in \mathbb{C}$.

Proof. Let $z_{0} \in \mathbb{C}$ such that $L\left(z_{0}\right)$ has simple spectrum. We may assume $L\left(z_{0}\right)$ is in diagonal form. Then $A=\operatorname{diag}\left(a_{1}, \ldots, a_{n}\right)$. Assume $a=a_{1}$ has multiplicity $r$ with $1 \leq r<n$ and $a=a_{1}=\ldots=a_{r}$. If $z \in \mathbb{C}$, then

$$
A L(z) e_{j}=L(z) A e_{j}=L(z) a e_{j}=a L(z) e_{j}, 1 \leq j \leq r,
$$

where $e_{j}$ is the $j$-th unit vector of $\mathbb{C}^{n}$. Hence $L(z) e_{j}$ is an eigenvector for the eigenvalue $a$ of $A$. This implies that

$$
L(z) e_{j}=\sum_{k=0}^{r} a_{j k}(z) e_{k}, 1 \leq j \leq r .
$$

So

$$
L(z)=\left[\begin{array}{cc}
A(z) & B(z) \\
0 & D(z)
\end{array}\right]
$$

and $\operatorname{det}(L(z)-\lambda)=\operatorname{det}(A(z)-\lambda) \operatorname{det}(D(z)-\lambda)$. This contradicts 3.1.2. 
Suppose $M \in \underline{\tilde{g}}_{w}$. Then $M$ can be written as $M=M_{+}+M_{0}+M_{-}$with $M_{+} \in z \underline{\tilde{g}}_{w,+}, M_{0} \in M_{n}(\mathbb{C})$ and $M_{-} \in z^{-1} \underline{\tilde{g}}_{w,-}$.

Lemma 3.4.3. Suppose $M \in \mathfrak{C}^{w}(L)$. Then $M \in \mathfrak{C}_{+}^{w}(L)+\mathfrak{C}_{-}^{w}(L)$ if and only if there exist $A, B \in M_{n}(\mathbb{C})$ such that $M_{+}-A, M_{-}-B \in \mathfrak{C}^{w}(L)$.

Proof. (a) The sufficiency is immediate.

(b) Let $A, B \in M_{n}(\mathbb{C})$ such that $M_{+}-A, M_{-}-B \in \mathfrak{C}^{w}(L)$.

Then $M=M_{+}+M_{0}+M_{-}, M_{+}-A+M_{-}-B \in \mathfrak{C}^{w}(L)$, which implies $M_{0}+A+B \in \mathfrak{C}^{w}(L)$. Furthermore, $M_{+}-A+\left(M_{0}+A+B\right) \in \mathfrak{C}_{+}^{w}(L)$ and $M_{-}-B \in \mathfrak{C}_{-}^{w}(L)$.

Thus $M_{+}-A+\left(M_{0}+A+B\right)+M_{-}-B=M \in \mathfrak{C}_{+}^{w}(L)+\mathfrak{C}_{-}^{w}(L)$.

Lemma 3.4.4. $\mathfrak{C}_{+}^{w}(L)+\mathfrak{C}_{-}^{w}(L)$ is closed in $\mathfrak{C}^{w}(L)$.

Proof. Let $M_{j} \in \mathfrak{C}_{+}^{w}(L)+\mathfrak{C}_{-}^{w}(L)$ be a sequence with $\lim _{j \rightarrow \infty} M_{j}=M$ in $\underline{\tilde{g}}_{w}$. By the previous lemma there exist $A_{j}, B_{j} \in M_{n}(\mathbb{C})$ such that $\left(M_{j}\right)_{+}-A_{j},\left(M_{j}\right)_{-}-B_{j} \in \mathfrak{C}^{w}(L)$. Then $\left[\left(M_{j}\right)_{+}, L\right]_{\tilde{g}}=\left[A_{j}, L\right]_{\underline{g}},\left[\left(M_{j}\right)_{-}, L\right]_{\underline{g}}=$ $\left[B_{j}, L\right]_{\underline{g}}$ and by assumption $\left(M_{j}\right)_{+} \rightarrow M_{+},\left(M_{j}\right)_{-} \rightarrow M_{-}$which implies the convergence of the sequences $\left[A_{j}, L\right]_{\underline{\tilde{g}}}$ and $\left[B_{j}, L\right]_{\underline{\tilde{g}}}$. Since $\left\{[C, L]_{\underline{g}} \mid C \in\right.$ $\left.M_{n}(\mathbb{C})\right\}$ is finite dimensional hence closed in $\underline{\tilde{g}}_{w}$, it follows

$$
\begin{gathered}
{\left[\left(M_{j}\right)_{+}, L\right]_{\underline{g}}=\left[A_{j}, L\right]_{\underline{g}} \rightarrow[A, L]_{\underline{g}}, \quad A \in M_{n}(\mathbb{C})} \\
{\left[\left(M_{j}\right)_{-}, L\right]_{\underline{g}}=\left[B_{j}, L\right]_{\underline{g}} \rightarrow[B, L]_{\underline{g}}, \quad B \in M_{n}(\mathbb{C}) .}
\end{gathered}
$$

Since $\left[A_{j}-A, L\right]_{\tilde{g}} \rightarrow 0$, one has by the above lemma $A_{j}-A \rightarrow a I, a \in \mathbb{C}$. Thus $A_{j} \rightarrow A+a I$. One obtains $\left(M_{j}\right)_{+}-A_{j} \rightarrow M_{+}-A-a I$. Similarly, $\left(M_{j}\right)_{-}-B_{j} \rightarrow M_{-}-B-b I, b \in \mathbb{C}$. Since $\mathfrak{C}^{w}(L)$ is closed, it follows $M_{+}-A-a I, M_{-}-B-b I \in \mathfrak{C}^{w}(L)$. Thus $M \in \mathfrak{C}_{+}^{w}(L)+\mathfrak{C}_{-}^{w}(L)$, by the previous lemma.

Set $\tilde{G}_{w}=G L_{n}\left(A_{w}\right)$. Denote by $\tilde{G}_{w,+}$ resp. $\tilde{G}_{w,-}$ the subgroups

$$
\begin{aligned}
& \tilde{G}_{w,+}=\left\{g \in \tilde{G}_{w} \mid g=\sum_{m \geq 0} g_{m} z^{m}\right\}, \\
& \tilde{G}_{w,-}=\left\{g \in \tilde{G}_{w} \mid g=\sum_{m \leq 0} g_{m} z^{m}\right\} .
\end{aligned}
$$


Let $\tilde{V}_{w,+}$ resp. $\tilde{V}_{w,-}$ be defined by

$$
\begin{aligned}
& \tilde{V}_{w,+}=\left\{g \in \tilde{G}_{w,+} \mid g=I+\sum_{m>0} g_{m} z^{m}\right\}, \\
& \tilde{V}_{w,-}=\left\{g \in \tilde{G}_{w,-} \mid g=I+\sum_{m<0} g_{m} z^{m}\right\} .
\end{aligned}
$$

By analogy with the definition of the centralizers $\mathfrak{C}^{w}(L), \mathfrak{C}_{ \pm}^{w}(L)$ we define

$$
\begin{gathered}
C^{w}(L)=\left\{g \in \tilde{G}_{w} \mid g L g^{-1}=L\right\} \\
C_{ \pm}^{w}(L)=C^{w}(L) \cap \tilde{G}_{w, \pm}
\end{gathered}
$$

Denote by $V \rightarrow e^{V}$ the exponential map from $\underline{\tilde{g}}_{w} \rightarrow \tilde{G}_{w}$. Define

$$
\Lambda=\left\{V \in \mathfrak{C}^{w}(L) \mid e^{V} \in C_{-}^{w}(L) C_{+}^{w}(L)\right\} .
$$

Theorem 3.4.5. $\Lambda$ is closed in $\mathfrak{C}^{w}(L)$.

In order to establish the theorem we show that $C_{-}^{w}(L) C_{+}^{w}(L)$ is closed in $C^{w}(L)$. From the following lemmas we will deduce the statement of the theorem.

Lemma 3.4.6. Suppose $g \in C^{w}(L)$. Assume furthermore, $g$ can be factored, $g=g_{-} g_{0} g_{+}$, with $g_{-} \in \tilde{V}_{w,-}, g_{0} \in G L_{n}(\mathbb{C})$ and $g_{+} \in \tilde{V}_{w,+}$. Then $g \in$ $C_{-}^{w}(L) C_{+}^{w}(L)$ if and only if there exist $a, b \in G L_{n}(\mathbb{C})$ such that $g_{-}^{-1} L g_{-}=$ $a^{-1} L a$ and $g_{+} L g_{+}^{-1}=b L b^{-1}$.

Proof. (a) The sufficiency follows immediately.

(b) Let $a, b \in G L_{n}(\mathbb{C})$ such that $g_{-}^{-1} L g_{-}=a^{-1} L a$ and $g_{+} L g_{+}^{-1}=b L b^{-1}$. Then

$$
a g_{-}^{-1}\left(g_{-} g_{0} g_{+}\right) g_{+}^{-1} b=a g_{0} b \in C^{w}(L) .
$$

Moreover,

$$
g_{-} a^{-1}\left(a g_{0} b\right)=g_{-} g_{0} b \in C_{-}^{w}(L), b^{-1} g_{+} \in C_{+}^{w}(L),
$$

which implies

$$
\left(g_{-} g_{0} b\right)\left(b^{-1} g_{+}\right)=g_{-} g_{0} g_{+} \in C_{-}^{w}(L) C_{+}^{w}(L) .
$$


Lemma 3.4.7. Let $\left\{g_{j}\right\}_{j=1}^{\infty}$ be a sequence in $C_{-}^{w}(L) C_{+}^{w}(L)$ such that $\lim _{j \rightarrow \infty} g_{j}=g$ exists and $g \in \tilde{V}_{w,-} G L_{n}(\mathbb{C}) \tilde{V}_{w,+}$. Then $g \in C_{-}^{w}(L) C_{+}^{w}(L)$.

Proof. (a) By 3.4.6, $g_{j} \in C_{-}^{w}(L) C_{+}^{w}(L)$ if and only if there exist $a_{j}, b_{j} \in$ $G L_{n}(\mathbb{C})$ such that $\left(g_{j}\right)_{-}^{-1} L\left(g_{j}\right)_{-}=a_{j}^{-1} L a_{j},\left(g_{j}\right)_{+} L\left(g_{j}\right)_{+}^{-1}=b_{j} L b_{j}^{-1}$. From

$$
\left(g_{j}\right)_{-}\left(g_{j}\right)_{0}\left(g_{j}\right)_{+} \rightarrow g_{-} g_{0} g_{+},
$$

it follows that $\left(g_{j}\right)_{-} \rightarrow g_{-},\left(g_{j}\right)_{-}^{-1} \rightarrow g_{-}^{-1}$. This implies $\left(g_{j}\right)_{-}^{-1} L\left(g_{j}\right)_{-}=$ $a_{j}^{-1} L a_{j} \rightarrow g_{-}^{-1} L g_{-}$.

(b) Since $\mathbb{P} M_{n}(\mathbb{C})$, the projective space of $M_{n}(\mathbb{C})$, is compact, $\left[a_{j}\right]$ has a subsequence $\left[a_{j_{k}}\right]$ in $\mathbb{P} M_{n}(\mathbb{C})$ which converges to $[a], a \in M_{n}(\mathbb{C})$. For the classical adjoint, $\mathfrak{A} \mathfrak{d}\left(a_{j_{k}}\right)$, we obtain $\left[\mathfrak{A} \mathfrak{d}\left(a_{j_{k}}\right)\right] \rightarrow[\mathfrak{A} \mathfrak{d}(a)]$ in $\mathbb{P} M_{n}(\mathbb{C})$.

(c) Denote by $\underline{\tilde{g}}_{w}^{p, q}$ the finite dimensional subspace of $\underline{\tilde{g}}_{w}$

$$
\underline{\tilde{g}}_{w}^{p, q}=\left\{P \in \underline{\tilde{g}}_{w} \mid P(z)=\sum_{j=-p}^{q} p_{j} z^{j}, p_{j} \in M_{n}(\mathbb{C}), j=-p, \ldots, q\right\} .
$$

The sequence $\left[a_{j}^{-1} L a_{j}\right]$ converges in $\mathbb{P} \underline{\tilde{g}}_{w}^{p, q}$. Moreover, $\left[\mathfrak{A} \mathfrak{d}\left(a_{j}\right) L a_{j}\right]$ converges in $\mathbb{P} \underline{\tilde{g}}_{w}^{p, q}$ and it follows

$$
\left[\mathfrak{A} \mathfrak{d}\left(a_{j_{k}}\right) L a_{j_{k}}\right] \rightarrow[\mathfrak{A} \mathfrak{d}(a) L a] \text { in } \mathbb{P} \tilde{g}_{w}^{p, q}
$$

Hence,

$$
\left[\mathfrak{A d}\left(a_{j}\right) L a_{j}\right] \rightarrow[\mathfrak{A} \mathfrak{d}(a) L a] \text { in } \mathbb{P} \underline{\tilde{g}}_{w}^{p, q}
$$

(d) From $[\mathfrak{A d}(a) L a]=\left[g_{-}^{-1} L g_{-}\right]$(by (a) and (c)) it follows that there exists $\alpha \in \mathbb{C}^{*}$ such that $\alpha\left(g_{-}^{-1} L g_{-}\right)=\mathfrak{A} \mathfrak{d}(a) L a$. We assert $a \in G L_{n}(\mathbb{C})$. Assume not. Then $\operatorname{det}\left(\alpha g_{-}^{-1} L g_{-}\right)=\alpha^{n} \operatorname{det} L=0$. Since $\operatorname{det} L$ is the coefficient of $\lambda^{0}$ in $f(z, \lambda)$, det $L=0$ yields a contradiction to the irreducibility of $f$. We obtain $\tilde{\alpha} g_{-}^{-1} L g_{-}=a^{-1} L a$, for $\tilde{\alpha} \in \mathbb{C}^{*}$.

(e) We assert $\tilde{\alpha}=1$. Consider

$$
\operatorname{tr}\left(\tilde{\alpha} g_{-}^{-1} L g_{-}\right)=\tilde{\alpha} \operatorname{tr} L=\operatorname{tr}\left(a^{-1} L a\right)=\operatorname{tr} L .
$$

We may assume $\operatorname{tr} L \neq 0$. Otherwise we can replace $L$ by $L+t \cdot I$ for some $t \in \mathbb{R}, t>>0$, since $L$ and $L+t \cdot I$ have the same centralizer. Thus $\tilde{\alpha}=1$ and $g_{-}^{-1} L g_{-}=a^{-1} L a$. By the previous lemma it follows that $g_{-} a^{-1} \in C^{w}(L)$. A similar argument shows $b^{-1} g_{+} \in C^{w}(L)$. So we conclude from 3.4.6 that $g=g_{-} g_{0} g_{+} \in C_{-}^{w}(L) C_{+}^{w}(L)$. 
Lemma 3.4.8. $C_{-}^{w}(L) C_{+}^{w}(L)$ is closed in $C^{w}(L)$.

Proof. Let $g_{j} \rightarrow g$, where $g_{j} \in C_{-}^{w}(L) C_{+}^{w}(L)$. Since $C^{w}(L)$ is closed, one obtains $g \in C^{w}(L)$. Then $g g_{j}^{-1} \rightarrow 1$, which implies that $g g_{j}^{-1} \in$ $\tilde{V}_{w,-} G L_{n}(\mathbb{C}) \tilde{V}_{w,+}$ for all $j \geq N$ for some $N \in \mathbb{N}$. Moreover, since $g \in C^{w}(L)$

$$
g g_{j}^{-1}=\left(g_{j}^{-1}\right)_{-} a_{j}^{-1}\left(a_{j}\left(g_{j}^{-1}\right)_{0} b_{j}\right) g b_{j}^{-1} \quad\left(g_{j}^{-1}\right)_{+} \in \tilde{V}_{w,-} G L_{n}(\mathbb{C}) \tilde{V}_{w,+},
$$

for some $a_{j}, b_{j} \in C L_{n}(\mathbb{C})$ by 3.4.6. Furthermore

$$
g \in a_{j}\left(a_{j}\left(g_{j}^{-1}\right)_{0} b_{j}\right)_{-}^{-1} \tilde{V}_{w,-} G L_{n}(\mathbb{C}) \tilde{V}_{w,+}\left(g_{j}^{-1}\right)_{+}^{-1} b_{j}
$$

since $G L_{n}(\mathbb{C})$ normalizes $\tilde{V}_{w,-}$ and $\tilde{V}_{w,+}$. From 3.4 .7 we conclude $g \in C_{-}^{w}(L) C_{+}^{w}(L)$.

Proof of Theorem 3.4.5. Let $V_{j} \in \Lambda$ such that $V_{j} \rightarrow V$. Then $e^{V_{j}} \rightarrow e^{V}$ with $e^{V_{j}} \in C_{-}^{w}(L) C_{+}^{w}(L)$. Since $C_{-}^{w}(L) C_{+}^{w}(L)$ is closed, it follows that $e^{V} \in C_{-}^{w}(L) C_{+}^{w}(L)$. Hence $V \in \Lambda$.

Suppose $L \in \underline{\tilde{g}}$. Denote by $\mathfrak{C}(L)$ the centralizer of $L$ in $\tilde{\tilde{g}}$ and by $\mathfrak{C}_{+}(L)$ resp. $\mathfrak{C}_{-}(L)$ the centralizer of $L$ in $\tilde{g} \otimes \mathbb{C}[z]$ resp. $\tilde{g} \otimes \mathbb{C}\left[z^{-1}\right]$. In the sequel we consider $\mathbb{C}\left[z, L_{0}\right]$ and $\mathbb{C}\left[z, L_{\infty}\right]$ to be contained in $M_{n}\left(\mathbb{C}\left[z, z^{-1}\right]\right)$.

Lemma 3.4.9. Suppose $L \in \underline{\tilde{g}}$ and that $f(z, \lambda)=\operatorname{det}(L(z)-\lambda)$ is irreducible. Then $\mathfrak{C}_{+}(L)$ resp. $\mathfrak{C}_{-}(L)$ is integral over $\mathbb{C}\left[z, L_{0}\right]$ resp. $\mathbb{C}\left[z^{-1}, L_{\infty}\right]$.

Proof. Since $f(z, \lambda)$ is irreducible the set $S$ of singular points of $X_{L} \subset \mathbb{P}^{2}(\mathbb{C})$ is a proper Zariski-closed subset of $X_{L}$, hence finite. Suppose $z_{0} \in \mathbb{C}$ is such that $I, L_{0}\left(z_{0}\right), \ldots, L_{0}^{n-1}\left(z_{0}\right)$ are linearly independent. Then there exist $\lambda_{0}, \ldots, \lambda_{n-1} \in M_{n}(\mathbb{C})^{*}$ such that $\operatorname{det}\left(\lambda_{j}\left(L_{0}^{k}\left(z_{0}\right)\right)\right)_{0 \leq j, k \leq n-1} \neq 0$. Denote by $\Lambda(z)$ the matrix

$$
\Lambda(z)=\left[\lambda_{j}\left(L_{0}^{k}(z)\right)_{0 \leq j, k \leq n-1}\right]
$$

Set

$$
U=\{z \in \mathbb{C} \mid \operatorname{det}(\Lambda(z)) \neq 0\} .
$$

Since $\operatorname{det}(\Lambda(z))$ is a polynomial and $\operatorname{det}(\Lambda(z)) \not \equiv 0$, it follows that $\mathbb{C}-U$ is finite. If $z \in U, M \in \mathfrak{C}_{+}(L)$, then

$$
M(z)=\sum_{k=0}^{n-1} c_{k}(z) L_{0}^{k}(z), \lambda_{j}(M(z))=\sum_{k=0}^{n-1} c_{k}(z) \lambda_{j}\left(L_{0}^{k}(z)\right), \quad j=0, \ldots, n-1 .
$$


Hence if $z \in U$, then

$$
\operatorname{det}(\Lambda(z))\left(c_{0}(z), \ldots, c_{n-1}(z)\right)^{T}=\mathfrak{A} \mathfrak{d}(\Lambda(z))\left(\lambda_{0}(M(z)), \ldots, \lambda_{n-1}(M(z))\right)^{T} .
$$

Set $u_{k}(z)=\operatorname{det}(\Lambda(z)) c_{k}(z), k=0, \ldots, n-1$. Then $u_{k}(z) \in \mathbb{C}[z]$ since $\mathfrak{A d}(\Lambda(z))$ and $\lambda_{j}(M(z))$ are polynomials on $U$. Now

$$
\operatorname{det}(\Lambda(z)) M(z)=\sum_{k=0}^{n-1} u_{k}(z) L_{0}^{k}(z)
$$

on $U$, hence everywhere on $\mathbb{C}$. Thus

$$
M(z) \in \mathbb{C}\left[z, L_{0}\right] \operatorname{det}(\Lambda(z))^{-1} I .
$$

Now $\mathbb{C}\left[z, L_{0}\right] \operatorname{det}(\Lambda(z))^{-1} I$ is a notherian $\mathbb{C}\left[z, L_{0}\right]$-module with $\mathfrak{C}_{+}(L)$ as $\mathbb{C}\left[z, L_{0}\right]$-submodule. This implies $\mathfrak{C}_{+}(L)$ is finitely generated over $\mathbb{C}\left[z, L_{0}\right]$, hence integral.

A similar argument shows that $\mathfrak{C}_{-}(L)$ is integral over $\mathbb{C}\left[z^{-1}, L_{\infty}\right]$.

Suppose $L \in \underline{\tilde{g}}$. We will call $L$ good if $f(z, \lambda)$ is irreducible, $X_{0} \cap X_{\infty}$ is nonsingular and $\mathfrak{C}_{+}(L)$ resp. $\mathfrak{C}_{-}(L)$ is the integral closure of $\mathbb{C}\left[z, L_{0}\right]$ resp. $\mathbb{C}\left[z^{-1}, L_{\infty}\right]$ in $M_{n}\left(\mathbb{C}\left[z, z^{-1}\right]\right)$, in symbols $\mathfrak{C}_{+}(L)=\overline{\mathbb{C}\left[z, L_{0}\right]}, \mathfrak{C}_{-}(L)=$ $\overline{\mathbb{C}\left[z^{-1}, L_{\infty}\right]}$.

Theorem 3.4.10. Suppose $L \in \underline{\tilde{g}}$ and also assume $L$ is good. Then

$$
H^{1}\left(X_{L}^{\prime}, \mathfrak{o}_{X_{L}^{\prime}}\right) \cong \mathfrak{C}(L) / \mathfrak{C}_{-}(L)+\mathfrak{C}_{+}(L)
$$

in particular $\operatorname{dim}\left(\mathfrak{C}(L) / \mathfrak{C}_{-}(L)+\mathfrak{C}_{+}(L)\right)=g\left(X_{L}^{\prime}\right)$.

Proof. Denote by $\mathfrak{U}^{\prime}=\left\{X_{0}^{\prime}, X_{\infty}^{\prime}\right\}$ the open, affine covering of $X_{L}^{\prime}$, where $p\left(X_{0}^{\prime}\right)=X_{0}$ and $p\left(X_{\infty}^{\prime}\right)=X_{\infty}$. We view $X_{L}^{\prime}$ as an analytic space and the coherent sheaf $\mathfrak{o}_{X_{L}^{\prime}}$ as coherent analytic sheaf and compute the cohomology group $H^{1}\left(\mathbb{Z}_{L}^{\prime}, o_{X_{L}^{\prime}}\right)$ in the analytic category via Čech cohomology with respect to the covering $\mathfrak{U}^{\prime}$. GAGA (Serre [23]) then establishes the isomorphism

$$
H^{1}\left(X_{L}^{\prime}, \mathfrak{o}_{X_{L}^{\prime}}\right) \cong H^{1}\left(\mathfrak{U}^{\prime}, \mathfrak{o}_{X_{L}^{\prime}}\right)
$$

The Čech complex has two terms:

$$
\begin{aligned}
& C^{0}=\Gamma\left(X_{0}^{\prime}, \mathfrak{o}_{X_{L}^{\prime}}\right) \oplus \Gamma\left(X_{\infty}^{\prime}, \mathfrak{o}_{X_{L}^{\prime}}\right), \\
& C^{1}=\Gamma\left(X_{0}^{\prime} \cap X_{\infty}^{\prime}, \mathfrak{o}_{X_{L}^{\prime}}\right),
\end{aligned}
$$


where $\Gamma$ denotes the global section functor. Now

$$
\begin{gathered}
\Gamma\left(X_{0}^{\prime}, \mathfrak{o}_{X_{L}^{\prime}}\right)=\mathfrak{o}_{X_{0}^{\prime}}=\overline{\mathbb{C}[z, \lambda] /\left(f_{0}\right)}, \\
\Gamma\left(X_{\infty}^{\prime}, \mathfrak{o}_{X_{L}^{\prime}}\right)=\mathfrak{o}_{X_{\infty}^{\prime}}=\overline{\mathbb{C}[z, \lambda] /\left(f_{\infty}\right)}, \\
\Gamma\left(X_{0}^{\prime} \cap X_{\infty}^{\prime}, \mathfrak{o}_{X_{L}^{\prime}}\right)=\mathfrak{o}_{X_{0}^{\prime} \cap X_{\infty}^{\prime}}=\overline{\mathbb{C}\left[z, z^{-1}, \lambda\right] /(f) \mid X_{0} \cap X_{\infty}} .
\end{gathered}
$$

The map $d: C^{0} \rightarrow C^{1}$ is given by addition in $C^{1}$. To compute $H^{1}\left(\mathfrak{U}^{\prime}, \mathfrak{o}_{X_{L}^{\prime}}\right)$ note that the image of $d, \operatorname{Im} d$, is the set of all expressions $k=k_{0}+k_{\infty}$ with $k_{0} \in \Gamma\left(X_{0}^{\prime}, \mathfrak{o}_{X_{L}^{\prime}}\right)$ and $k_{\infty} \in \Gamma\left(X_{\infty}^{\prime}, \mathfrak{o}_{X_{L}^{\prime}}\right)$.

Since $L$ is good the following maps

$$
\begin{array}{r}
\gamma: \mathbb{C}\left[z, z^{-1}, \lambda\right] /(f) \mid X_{0} \cap X_{\infty} \rightarrow \mathbb{C}\left[z, z^{-1}, L\right] /(f) \\
z \rightarrow z, z^{-1} \rightarrow z^{-1}, \lambda \rightarrow L, \\
\gamma_{+}: \mathbb{C}[z, \lambda] /\left(f_{0}\right) \mid X_{0} \rightarrow \mathbb{C}\left[z, L_{0}\right] /\left(f_{0}\right) \\
z \rightarrow z, \lambda \rightarrow L_{0}=z^{p} L \\
\gamma_{-}: \mathbb{C}\left[z^{-1}, \lambda\right] /\left(f_{\infty}\right) \mid X_{\infty} \rightarrow \mathbb{C}\left[z, L_{\infty}\right] /\left(_{\infty}\right) \\
z^{-1} \rightarrow z^{-1}, \lambda \rightarrow L_{\infty}=z^{-q} L
\end{array}
$$

establish the isomorphisms

$$
\begin{array}{r}
\Gamma\left(X_{0}^{\prime} \cap X_{\infty}^{\prime}, \mathfrak{o}_{X_{L}^{\prime}}\right) \cong \mathfrak{C}(L), \Gamma\left(X_{0}^{\prime}, \mathfrak{o}_{X_{L}^{\prime}}\right) \cong \mathfrak{C}_{-}(L) \\
\Gamma\left(X_{0}^{\prime}, \mathfrak{o}_{X_{L}^{\prime}}\right) \cong \mathfrak{C}_{+}(L) .
\end{array}
$$

Thus $H^{1}\left(\mathfrak{U}^{\prime}, \mathfrak{o}_{X_{L}^{\prime}}\right)$

$$
\begin{aligned}
H^{1}\left(\mathfrak{U}^{\prime}, \mathfrak{o}_{X_{L}^{\prime}}\right) & \cong \Gamma\left(X_{0}^{\prime} \cap X_{\infty}^{\prime}, \mathfrak{o}_{X_{L}^{\prime}}\right) / \Gamma\left(X_{\infty}^{\prime}, \mathfrak{o}_{X_{L}^{\prime}}\right)_{\mid X_{0}^{\prime} \cap X_{\infty}^{\prime}}+\Gamma\left(X_{0}^{\prime}, \mathfrak{o}_{X_{L}^{\prime}}\right)_{\mid X_{0}^{\prime} \cap X_{\infty}^{\prime}} \\
& \cong \mathfrak{C}(L) / \mathfrak{C}_{-}(L)+\mathfrak{C}_{+}(L) .
\end{aligned}
$$

Lemma 3.4.11. Suppose $L \in \underline{\tilde{g}}$ and also assume that $f(z, \lambda)$ is irreducible and $X_{0} \cap X_{\infty}$ is nonsingular. If in addition $\operatorname{dim}\left(\mathfrak{C}(L) / \mathfrak{C}_{-}(L)+\mathfrak{C}_{+}(L)\right)=$ $g\left(X_{L}^{\prime}\right)$, then $L$ is good.

Proof. Since $X_{0} \cap X_{\infty}$ is nonsingular we have the isomorphism

$$
\Gamma\left(X_{0}^{\prime} \cap X_{\infty}^{\prime}, \mathfrak{o}_{X_{L}^{\prime}}\right)=\Gamma\left(X_{0} \cap X_{\infty}, \mathfrak{o}_{X_{L}}\right) \cong \mathfrak{C}(L) .
$$


From the irreducibility of $f(z, \lambda)$ we obtain that $\mathfrak{C}_{+}(L)$ resp. $\mathfrak{C}_{-}(L)$ is integral over $\mathbb{C}\left[z, L_{0}\right]$ resp. $\mathbb{C}\left[z^{-1}, L_{\infty}\right]$ and

$$
\begin{aligned}
\mathbb{C}\left[z, L_{0}\right] \subseteq \mathfrak{C}_{+}(L) \subseteq \overline{\mathbb{C}\left[z, L_{0}\right]} \\
\mathbb{C}\left[z, L_{\infty}\right] \subseteq \mathfrak{C}_{-}(L) \subseteq \overline{\mathbb{C}\left[z, L_{\infty}\right]}
\end{aligned}
$$

Assume $\mathfrak{C}_{+}(L) \neq \overline{\mathbb{C}\left[z, L_{0}\right]}$ or $\mathfrak{C}_{-}(L) \neq \overline{\mathbb{C}\left[z^{-1}, L_{\infty}\right]}$. Then $\mathfrak{C}_{+}(L), \mathfrak{C}_{-}(L)$ define a curve $\tilde{X}_{L}$ with

$$
X_{L}^{\prime} \rightarrow \tilde{X}_{L} \stackrel{\tilde{p}}{\rightarrow} X_{L}
$$

and $\mathfrak{C}_{+}(L) \cong \mathfrak{o}_{\tilde{X}_{0}}, \mathfrak{C}_{-}(L) \cong \mathfrak{o}_{\tilde{X}_{\infty}}, \tilde{p}\left(\tilde{X}_{0}\right)=X_{0}, \tilde{p}\left(\tilde{X}_{\infty}\right)=X_{\infty}, \quad \tilde{X}_{0} \cap \tilde{X}_{\infty}=$ $X_{0} \cap X_{\infty}$. Thus

$$
\begin{aligned}
g\left(X_{L}^{\prime}\right)=\operatorname{dim} H^{1}\left(X_{L}^{\prime}, \mathfrak{o}_{X_{L}^{\prime}}\right) & <\operatorname{dim} H^{1}\left(\tilde{X}_{L}, \mathfrak{o}_{\tilde{X}_{L}}\right) \\
& =\operatorname{dim}\left(\mathfrak{C}(L) / \mathfrak{C}_{-}(L)+\mathfrak{C}_{+}(L)\right) .
\end{aligned}
$$

(Cf. Serre [24], p. 73.)

Theorem 3.4.12. Suppose $L \in \underline{\tilde{g}}$ is as in 3.1 .5 or 3.1.6. Then

$$
\begin{gathered}
\operatorname{dim}\left(\mathfrak{C}^{w}(L) / \mathfrak{C}_{-}^{w}(L)+\mathfrak{C}_{+}^{w}(L)\right)=g\left(X_{L}^{\prime}\right) \text { and } \\
\mathfrak{C}^{w}(L) / \mathfrak{C}_{-}^{w}(L)+\mathfrak{C}_{+}^{w}(L) \cong \mathfrak{C}(L) / \mathfrak{C}_{+}(L)+\mathfrak{C}_{+}(L) .
\end{gathered}
$$

In particular $L$ is good.

Proof. (a) (nonsingular case). Consider $L_{0}(z)=z^{p} L(z)$. Then the condition on the nonsingularity of $f(z, \lambda)$ implies that $I, L_{0}(z), \ldots, L_{0}^{n-1}(z)$ are linearly independent for all $z \in \mathbb{C}$. For the same reason one has that $I, L_{\infty}\left(z^{-1}\right), \ldots, L_{\infty}^{n-1}\left(z^{-1}\right)$ are linearly independent for all $z^{-1} \in \mathbb{C}$. From the characterization of $\mathfrak{C}^{w}(L), \mathfrak{C}_{ \pm}^{w}(L)$ (3.4.1) it follows if $M \in \mathfrak{C}^{w}(L)$

$$
\begin{aligned}
M(z) & =\sum_{j=0}^{n-1} c_{j}(z) L^{j}(z), c_{j}(z) \in A_{w} \\
& =\sum_{j=0}^{n-1}\left(\sum_{k<-l} a_{j, k} z^{k}+\sum_{k \geq-l}^{m} a_{j, k} z^{k}+\sum_{k>m} a_{j, k} z^{k}\right) L^{j}(z)
\end{aligned}
$$

for $l, m \in \mathbb{N}$. Thus the set

$$
\left\{z^{k} L^{j}(z) \mid 1 \leq j \leq n-1,-j p+1 \leq k \leq j q-1\right\}
$$


provides a basis for $\mathfrak{C}^{w}(L) / \mathfrak{C}_{+}^{w}(L)+\mathfrak{C}_{-}^{w}(L)$. A counting argument shows that the dimension equals

$$
\sum_{j=1}^{n-1}(j(p+q)-1)=\frac{1}{2} n(n-1)(p+q)-n+1=g\left(X_{L}\right) .
$$

(b) (tridiagonal case). Consider $L_{0}(z)=\ell_{0}+z \ell_{1}+z^{2} \ell_{2}$. For the proof of the theorem in the tridiagonal case we will need the following scholia.

Scholium I. If $0 \leq j \leq n-2$, then $\ell_{0} \ell_{1}^{j} \ell_{0}=0$ and $\ell_{2} \ell_{1}^{j} \ell_{2}=0$.

Proof. We assert that $\ell_{0} \ell_{1}^{j} E_{r, n}=0$ if $j+r \leq n-1$. We prove this by induction on $j$. If $j=0$, then $\ell_{0} E_{r, n}=E_{1, n} E_{r, n}=0, r<n$. Assume the inductive hypothesis for $j$. Then

$$
\ell_{1} E_{r, n}=a_{r-1} E_{r-1, n}+d_{r} E_{r, n}+b_{r} E_{r+1, n} .
$$

Thus

$$
\ell_{0} \ell_{1}^{j+1} E_{r, n}=a_{r-1} \ell_{0} \ell_{1}^{j} E_{r-1, n}+d_{r} \ell_{0} \ell_{1}^{j} E_{r, n}+b_{r} \ell_{0} \ell_{1}^{j} E_{r+1, n} .
$$

If $j+1+r<n$, then $j+r-1<n, j+r<n$ and $j+r+1<n$. Thus the inductive hypothesis implies the result for $j+1$. The Scholium is just the assertion for $r=1$.

By applying the anti-automorphism $x \rightarrow x^{T}$ one obtains $\ell_{2} \ell_{1}^{j} \ell_{2}=0$ for $0 \leq j \leq n-2$.

Scholium II. If $1 \leq j \leq n-1$, then $L_{0}^{j}(z)=z^{j-1} M_{j}(z), M_{j}(z) \in$ $\mathfrak{B}\left(z, M_{n}(\mathbb{C})\right)$, i.e. $M_{j}(z)$ is polynomial.

Proof. This result is clear for $j=1$. Assume that we have shown that

$$
L_{0}^{j}(z)=z^{j-1} M_{j}(z)=z^{j-1}\left(A_{j, j-1}+z A_{j, j}+\ldots\right)
$$

and $\ell_{0} A_{j, j-1}=0$. We show that

$$
L_{0}^{j+1}(z)=z^{j} A_{j+1, j}+z^{j+1} A_{j+1, j+1}+\ldots
$$

with $\ell_{0} A_{j+1, j}=0$ for $j+1 \leq n-1$.

$$
\begin{aligned}
L_{0}^{j+1}(z) & =\ell_{0} L_{0}^{j}(z)+z \ell_{1} L_{0}^{j}(z)+z^{2} \ell_{2} L_{0}^{j}(z) \\
& =\ell_{0}\left(z^{j-1} A_{j, j-1}+z^{j} A_{j, j}+\ldots\right) \\
& +\ell_{1}\left(z^{j} A_{j, j-1}+z^{j+1} A_{j, j}+\ldots\right) \\
& +\ell_{2}\left(z^{j+1} A_{j, j-1}+z^{j+2} A_{j, j}+\ldots\right) .
\end{aligned}
$$


Now $\ell_{0} A_{j, j-1}=0$ by assumption. Thus

$$
L_{0}^{j+1}(z)=z^{j}\left(\ell_{0} A_{j, j}+\ell_{1} A_{j, j-1}\right)+z^{j+1}\left(\ell_{1} A_{j, j}+\ell_{2} A_{j, j-1}\right)+\ldots
$$

Hence

$$
A_{j+1, j}=\ell_{0} A_{j, j}+\ell_{1} A_{j, j-1} .
$$

But then $\ell_{0} A_{j+1, j}=\ell_{0} \ell_{1} A_{j, j-1}$. Applying equation (3) to $A_{j, j-1}$ we find

$$
\begin{aligned}
\ell_{0} A_{j+1, j} & =\ell_{0} \ell_{1} \ell_{0} A_{j-1, j-1}+\ell_{0} \ell_{1}^{2} A_{j-1, j-2} \\
& =\ell_{0} \ell_{1}^{2} A_{j-1, j-2} .
\end{aligned}
$$

We continue to apply equation (3) and finally get

$$
\begin{aligned}
\ell_{0} A_{j+1, j} & =\ell_{0} \ell_{1}^{j-2} A_{2,1}=\ell_{0} \ell_{1}^{j-2}\left(\ell_{1} \ell_{0}+\ell_{0} \ell_{1}\right) \\
& =\ell_{0} \ell_{1}^{j-1} \ell_{0}+\ell_{0} \ell_{1}^{j-2} \ell_{0} \ell_{1}=0, \text { if } j+1 \leq n-1
\end{aligned}
$$

by Scholium I. The statement now follows.

Scholium III. If $\prod_{j=1}^{n-1} b_{j} \neq 0, \ell_{0} \neq 0$, then $I, M_{1}(0), \ldots, M_{n-1}(0)$ are linearly independent.

Proof. Since $M_{j+1}(0)=A_{j+1, j}$ and $A_{j+1, j}=\ell_{0} A_{j, j}+\ell_{1} A_{j, j-1}, 0 \leq j \leq n-2$, it is enough to show that $I, \ell_{0}, \ell_{1} \ell_{0}, \ldots, \ell_{1}^{n-2} \ell_{0}$ are linearly independent. We assert

$$
\ell_{1}^{j} \ell_{0}=\sum_{k=0}^{j} c_{k} E_{k+1, n}, 0 \leq j \leq n-2
$$

with $c_{j}=\prod_{k=1}^{j} b_{k}$. We proceed by induction. The result is obvious for $j=0$. Assume the inductive hypothesis for $j$. Then

$$
\begin{aligned}
\ell_{1}^{j+1} \ell_{0} & =\ell_{1}\left(\ell_{1}^{j} \ell_{0}\right)=\ell_{1}\left(\sum_{k=0}^{j} c_{k} E_{k+1, n}\right) \\
& =\sum_{k=0}^{j} c_{k} \ell_{1} E_{k+1, n} \\
& =\sum_{k=0}^{j-1} \tilde{c}_{k} E_{k+1, n}+c_{j}\left(a_{j} E_{j, n}+d_{j+1} E_{j+1, n}+b_{j+1} E_{j+2, n}\right) \\
& =\sum_{k=0}^{j+1} \tilde{\tilde{c}}_{k} E_{k+1, n},
\end{aligned}
$$


where $\tilde{\tilde{c}}_{j+1}=c_{j} b_{j+1}=\prod_{k=1}^{j+1} b_{k}$. It follows that

$$
\ell_{1}^{j+1} \equiv E_{j+2, n} \bmod \left(\mathbb{C} E_{1, n}+\ldots+\mathbb{C} E_{j+1, n}\right)
$$

which implies that $\ell_{0}, \ell_{1} \ell_{0}, \ldots, \ell_{1}^{n-2} \ell_{0}$ are linearly independent. Consequently $I, \ell_{0}, \ell_{1} \ell_{0}, \ldots, \ell_{1}^{n-2} \ell_{0}$ are linearly independent.

Consider $L_{\infty}\left(z^{-1}\right)=z^{-2} \ell_{0}+z^{-1} \ell_{1}+\ell_{2}$. By the same chain of arguments one shows that $L_{\infty}^{j}\left(z^{-1}\right)=z^{-j+1} \overline{M_{j}\left(z^{-1}\right)}$, with $\overline{M_{j}\left(z^{-1}\right)} \in \mathfrak{B}\left(z^{-1}, M_{n}(\mathbb{C})\right)$. If in addition $\prod_{j=1}^{n-1} a_{j} \neq 0$, then $I, \overline{M(0)}, \ldots, \overline{M_{n-1}(0)}$ are linearly independent. The relation between $M_{j}(z)$ and $\overline{M_{j}\left(z^{-1}\right)}$ is as follows:

$$
\begin{aligned}
\overline{M_{j}\left(z^{-1}\right)} & =z^{j-1} L_{\infty}^{j}\left(z^{-1}\right)=z^{j-1}\left(z^{-2 j} L_{0}^{j}(z)\right) \\
& =z^{-j-1} L_{0}^{j}(z)=z^{-j-1}\left(z^{j-1} M_{j}(z)\right) \\
& =z^{-2} M_{j}(z), \quad 1 \leq j \leq n-1
\end{aligned}
$$

If $M(z) \in \mathfrak{C}^{w}(L)$, then by 3.4.1 and Scholium III

$$
M(z)=\sum_{j=1}^{n-1} \alpha_{j}(z) M_{j}(z), \quad\left(M_{0}(z) \equiv I\right), \quad \alpha_{j}(z) \in A_{w} .
$$

Write

$$
\begin{aligned}
\alpha_{j}(z) & =\alpha_{j}^{+}(z)+\alpha_{j}^{-}(z) \\
\alpha_{j}^{+}(z) & =\sum_{k \geq 0} \alpha_{j, k} z^{k}, \quad \alpha_{j}^{-}(z)=\sum_{k<0} \alpha_{j, k} z^{k} .
\end{aligned}
$$

Then

$$
\sum_{j=0}^{n-1} \alpha_{j}^{+}(z) M_{j}(z) \in \mathfrak{C}_{+}^{w}(L)
$$

and if $\beta(z)=\sum_{k \leq-2} \alpha_{j, k} z^{k}$, then

$$
\sum_{j=1}^{n-1} \beta_{\jmath}(z) M_{j}(z)+\alpha_{0}(z) I \in \mathfrak{C}_{-}^{w}(L) .
$$

Thus

$$
M(z)=\sum_{j=1}^{n-1} \alpha_{j,-1} z^{-1} M_{j}(z) \quad \bmod \mathfrak{C}_{-}^{w}(L)+\mathfrak{C}_{+}^{w}(L)
$$


So $\operatorname{dim}\left(\mathfrak{C}^{w}(L) / \mathfrak{C}_{-}^{w}(L)+\mathfrak{C}_{+}^{w}(L)\right) \leq n-1$. Now

$$
\begin{aligned}
& \mathfrak{C}_{+}^{w}(L)=\left\{\sum_{j=1}^{n-1} \gamma_{j}(z) M_{j}(z) \mid \gamma_{j}(z)=\sum_{k \geq 0} \gamma_{j, k} z^{k}, \gamma_{j}(z) \in A_{w}, 1 \leq j \leq n-1\right\}, \\
& \mathfrak{C}_{-}^{w}(L)=\left\{\sum_{j=1}^{n-1} \gamma_{j}(z) \overline{M_{j}(z)} \mid \gamma_{j}(z)=\sum_{k \leq 0} \gamma_{j, k} z^{k}, \gamma_{j}\left(z^{-1}\right) \in A_{w}, 1 \leq j \leq n-1\right\} .
\end{aligned}
$$

Thus if

$$
\sum_{j=1}^{n-1} c_{\jmath} z^{-1} M_{j}(z) \in \mathfrak{C}_{-}^{w}(L)+\mathfrak{C}_{+}^{w}(L),
$$

then by equation (4)

$$
\sum_{j=1}^{n-1} c_{j} z^{-1} M_{j}(z)=\sum_{j=1}^{n-1} \gamma_{j}(z) M_{j}(z)+\sum_{j=1}^{n-1} \delta_{j}\left(z^{-1}\right) z^{-2} M_{j}(z) .
$$

So $c_{j} z^{-1}=\gamma_{j}(z)+\delta_{j}\left(z^{-1}\right) z^{-2}, z \neq 0$. This implies $c_{j}=0$ for all $1 \leq j \leq n-1$. Thus $\operatorname{dim}\left(\mathfrak{C}^{w}(L) / \mathfrak{C}_{-}^{w}(L)+\mathfrak{C}_{+}^{w}(L)\right)=n-1$.

(c) From the proof of (a) and (b) it follows immediately that

$$
\mathfrak{C}^{w}(L) / \mathfrak{C}_{-}^{w}(L)+\mathfrak{C}_{+}^{w}(L) \cong \mathfrak{C}(L) / \mathfrak{C}_{-}(L)+\mathfrak{C}_{+}(L) .
$$

Theorem 3.4.13. Suppose $L \in \underline{\tilde{g}}$ is good. Then

$$
\operatorname{Jac}\left(X_{L}^{\prime}\right) \cong \mathfrak{C}^{w}(L) / \mathfrak{C}_{-}^{w}(L)+\mathfrak{C}_{+}^{w}(L) / \Lambda / \mathfrak{C}_{-}^{w}(L)+\mathfrak{C}_{+}^{w}(L) \cong \mathfrak{C}^{w}(L) / \Lambda .
$$

Proof. We have (cf. Gunning [11], pp. 130, 152, 157)

$$
\operatorname{Jac}\left(X_{L}^{\prime}\right) \cong H^{1}\left(X_{L}^{\prime}, \mathfrak{o}_{X_{L}^{\prime}}\right) / H^{1}\left(X_{L}^{\prime}, \mathbb{Z}\right) .
$$

Now $H^{1}\left(X_{L}^{\prime}, \mathfrak{o}_{X_{L}^{\prime}}\right) \cong H^{1}\left(\mathfrak{U}^{\prime}, \mathfrak{o}_{X_{L}^{\prime}}\right)$ by GAGA. The short exact sequence

$$
0 \rightarrow \mathbb{Z} \rightarrow \mathfrak{o}_{X_{L}^{\prime}} \stackrel{e}{\rightarrow} \mathfrak{o}_{X_{L}^{\prime}}^{*} \rightarrow 0
$$

induces a surjective homomorphism

$$
H^{1}\left(\mathfrak{U}^{\prime}, \mathfrak{o}_{X_{L}^{\prime}}\right) \rightarrow H^{1}\left(\mathfrak{U}^{\prime}, \mathfrak{o}_{X_{L}^{\prime}}\right) / H^{1}\left(\mathfrak{U}^{\prime}, \mathbb{Z}\right)
$$

from which we obtain a surjective homomorphism (notation in 3.4.10).

$$
\Gamma\left(X_{0}^{\prime} \cap X_{\infty}^{\prime}, \mathfrak{o}_{X_{L}^{\prime}}\right) \rightarrow H^{1}\left(\mathfrak{U}^{\prime}, \mathfrak{o}_{X_{L}^{\prime}}\right) / H^{1}\left(\mathfrak{U}^{\prime}, \mathbb{Z}\right) .
$$


The kernel $K$ of this map consists of all functions $k$ such that $e^{k}=e^{k_{0}} e^{k_{\infty}}$, with $k_{0} \in \Gamma\left(X_{0}^{\prime}, \boldsymbol{o}_{X_{L}^{\prime}}\right)_{\left.\right|_{X_{0} \cap X_{\infty}}}$ and $k_{\infty} \in \Gamma\left(X_{\infty}^{\prime}, \boldsymbol{o}_{X_{L}^{\prime}}\right)_{\left.\right|_{X_{0} \cap x_{\infty}}}$. If $\tilde{\Lambda}$ denotes the analogue of $\Lambda$ in $\underline{\tilde{g}}$, then (notation in 3.4.10)

$$
K / \operatorname{Im} d \cong \tilde{\Lambda} / \mathfrak{C}_{-}(L)+\mathfrak{C}_{+}(L) .
$$

Hence

$$
\begin{aligned}
& \mathfrak{C}^{w}(L) / \mathfrak{C}_{-}^{w}(L)+\mathfrak{C}_{+}^{w}(L) / \Lambda / \mathfrak{C}_{-}^{w}(L)+\mathfrak{C}_{+}^{w}(L) \\
& \cong \mathfrak{C}(L) / \mathfrak{C}_{-}(L)+\mathfrak{C}_{+}(L) / \tilde{\Lambda} / \mathfrak{C}_{-}(L)+\mathfrak{C}_{+}(L) \\
& \cong \Gamma\left(X_{0}^{\prime} \cap X_{\infty}^{\prime}, \mathfrak{o}_{X_{L}^{\prime}}\right) / \operatorname{Im} d / K / \operatorname{Im} d \\
& \cong \Gamma\left(X_{0}^{\prime} \cap X_{\infty}^{\prime}, \mathfrak{o}_{X_{L}^{\prime}}\right) / K \\
& \cong H^{1}\left(\mathfrak{U}^{\prime}, \mathfrak{o}_{X_{L}^{\prime}}\right) / H^{1}\left(\mathfrak{U}^{\prime}, \mathbb{Z}\right) \\
& \cong H^{1}\left(X_{L}^{\prime}, \mathfrak{o}_{X_{L}^{\prime}}\right) / H^{1}\left(\mathfrak{U}^{\prime}, \mathbb{Z}\right) .
\end{aligned}
$$

Since by GAGA we also obtain $H^{1}\left(X_{L}^{\prime}, \mathfrak{o}_{X_{L}^{\prime}}^{*}\right) \cong H^{1}\left(\mathfrak{U}^{\prime}, \mathfrak{o}_{X_{L}^{\prime}}^{*}\right)$, we can replace the exact sequence

$$
0 \rightarrow H^{1}\left(\mathfrak{U}^{\prime}, \mathbb{Z}\right) \rightarrow H^{1}\left(\mathfrak{U}^{\prime}, \mathfrak{o}_{X_{L}^{\prime}}\right) \rightarrow H^{1}\left(\mathfrak{U}^{\prime}, \mathfrak{o}_{X_{L}^{\prime}}^{*}\right) \rightarrow H^{2}\left(\mathfrak{U}^{\prime}, \mathbb{Z}\right) \rightarrow 0
$$

by the exact sequence

$$
0 \rightarrow H^{1}\left(\mathfrak{U}^{\prime}, \mathbb{Z}\right) \rightarrow H^{1}\left(X_{L}^{\prime}, \mathfrak{o}_{X_{L}^{\prime}}\right) \rightarrow H^{1}\left(X_{L}^{\prime}, \mathfrak{o}_{X_{L}^{\prime}}^{*}\right) \rightarrow H^{1}\left(\mathfrak{U}^{\prime}, \mathbb{Z}\right) \rightarrow 0 .
$$

It follows that $\operatorname{Jac}\left(X_{L}^{\prime}\right) \cong H^{1}\left(X_{L}^{\prime}, \mathfrak{o}_{X_{L}^{\prime}}\right) / H^{1}\left(\mathfrak{U}^{\prime}, \mathbb{Z}\right)$. Hence

$$
\operatorname{Jac}\left(X_{L}^{\prime}\right) \cong \mathfrak{C}^{w}(L) / \mathfrak{C}_{-}^{w}(L)+\mathfrak{C}_{+}^{w}(L) / \Lambda / \mathfrak{C}_{-}^{w}(L)+\mathfrak{C}_{+}^{w}(L) .
$$

\subsection{Theta Functions as Matrix Entries of the Standard Represen- tations of the Basic Module.}

In this section we want to employ the basic representation $\left(L\left(\lambda_{0}\right), \pi_{\lambda_{0}}\right)$ of $\widehat{S L}_{n}\left(A_{w}\right)$ and the method of representative functions to obtain theta functions. The map $X \rightarrow X-n^{-1} \operatorname{tr}(X) \cdot I$ is a continuous projection from $M_{n}\left(A_{w}\right)$ onto $\underline{s l}_{n}\left(A_{w}\right)$. Its restriction to the quotient $\mathfrak{C}^{w}(L) / \mathfrak{C}_{-}^{w}(L)+$ $\mathfrak{C}_{+}^{w}(L)$ is for $L \in M_{n}\left(A_{w}\right), L(z)=\sum_{j=-p}^{q} \ell_{j} z^{j}$, injective, since $A_{w} \cdot I \subset$ $\mathfrak{C}_{-}^{w}(L)+\mathfrak{C}_{+}^{w}(L)$. We thus may assume without loss of generality $\underline{g}=$ $\underline{s l}_{n}(\mathbb{C}), G=S L_{n}(\mathbb{C})$ and $L \in \underline{\tilde{g}}$. We suppose in addition that $L$ is good. 
Another way to employ the basic representation is to induce it to $M_{n}\left(A_{w}\right)$ by letting $A_{w} \cdot I$ act as $O$ on $L\left(\lambda_{0}\right)$. On the level of the group we consider the simply connected covering $\mathbb{C}^{*}\left(S L_{n}(\mathbb{C})\right)$ of $G L_{n}(\mathbb{C})$ and let $\mathbb{C}^{*} \operatorname{Center}\left(S L_{n}(\mathbb{C})\right)$ act by 1 on the module. In order to obtain the same results one could also use the construction of the universal central extension of $\widetilde{G L}_{n}(\mathbb{C})$ and its action on the dual determinant bundle of some Grassmanian manifold. For details we refer to Pressley-Segal [19] and Segal-Wilson [22].

We use the notation exp for the exponential map from $\underline{\hat{g}}_{w}$ to $\hat{G}_{w}$ and $e^{x}$ for the exponential map from $\underline{\tilde{g}}_{w}$ to $\tilde{G}_{w}$. Let pr be the projection pr: $\hat{G}_{w} \rightarrow \tilde{G}_{w}$. We note that

$$
\text { pr }: \hat{V}_{w,-} G \hat{V}_{w,+} \rightarrow \tilde{V}_{w,-} G \tilde{V}_{w,+}
$$

is an analytic diffeomorphism with kernel $\left\{e^{t c} \mid t \in \mathbb{C}\right\}$. Here, $\hat{V}_{w, \pm}$ denote the images of $\tilde{V}_{w, \pm}$ in $\hat{G}_{w}$. On $\hat{V}_{w,-} G \hat{V}_{w,+}$ the exact sequence $1 \rightarrow$ $\mathbb{C}^{*} \rightarrow \hat{G}_{w} \rightarrow \tilde{G}_{w} \rightarrow 1$ splits and we denote by $\psi$ the cross-section $\psi: \tilde{V}_{w,-} G \tilde{V}_{w,+} \rightarrow \hat{V}_{w,-} G \hat{V}_{w,+}$ (Wallach [Lecture Notes]).

Suppose $V \in \Lambda$. Then $e^{V}$ can be represented as

$$
e^{V}=g_{-}(V) g_{+}(V), g_{ \pm}(V) \in C_{ \pm}^{w}(L) .
$$

If $e^{V}=h_{-}(V) h_{+}(V)$ with $h_{ \pm}(V) \in C_{ \pm}^{w}(L)$, then by $3.4 .2 h_{+}(V)^{-1} g_{+}(V)=$ $a I$ with $a I \in G$. Assume $V \in \underline{g}_{w}$ such that $\exp V \in \hat{V}_{w,-} G \hat{V}_{w,+}$. Then $\exp V$ can be expressed as follows:

3.5.1.

$$
\exp V=\psi\left(g_{-}(V) g_{+}(V)\right) e^{\gamma(V) c}
$$

where $\gamma$ is a function $\gamma: \underline{\tilde{g}}_{w} \rightarrow \mathbb{C}$.

Lemma 3.5.2. If $X, Y \in \underline{\tilde{g}}_{w}$ and $[X, Y]_{\underline{g}}=0$, then

$$
\exp (X) \exp (Y)=\exp (X+Y) e^{\frac{1}{2} \omega(X, Y) c} .
$$

Proof. Assume $r, s \in \mathbb{C}$ are small. Since $[X, Y]_{\underline{g}}=0$

$$
\exp (r X) \exp (s Y) \exp (-(r X+s Y))=e^{\Phi(r X, s Y) c},
$$

where $\Phi(r X, s Y)$ is a holomorphic function in $r$ and $s$. Thus we may assume that $\|X\|_{w},\|Y\|_{w}$ are small, such that we can apply the Campbell-Hausdroff 
formula. It follows

$$
\begin{aligned}
\exp (X) \exp (Y) & =\exp \left(X+Y+\frac{1}{2}[X, Y]+\text { higher commutators }\right) \\
& =\exp \left(X+Y+\frac{1}{2}[X, Y]\right)
\end{aligned}
$$

since $c$ is central and $[X, Y]_{\underline{\tilde{g}}}=0$ by hypothesis. Now $[X, Y]=[X, Y]_{\underline{\tilde{g}}}+$ $\omega(X, Y) c$. Hence

$$
\exp (X) \exp (Y)=\exp (X+Y) e^{\frac{1}{2} \omega(X, Y) c} .
$$

Corollary. Under the assumption of the lemma

$$
\exp (X+Y)=\exp (X) \exp (Y) e^{-\frac{1}{2} \omega(X, Y) c} .
$$

Suppose $v_{0} \in L\left(\lambda_{0}\right)$ is a highest weight vector of the basic module $L\left(\lambda_{0}\right)$. Suppose $W \in \mathfrak{C}^{w}(L)$. Define the function $\Phi$ as follows:

$$
\Phi(W)=\left\langle\pi_{\lambda_{0}}(\exp W) v_{0}, v_{0}\right\rangle_{\lambda_{0}} .
$$

Denote by $\operatorname{Ad}_{\underline{g}_{w}}$ resp. $\operatorname{Ad}_{\underline{g}_{w}}$ the adjoint representation of $\hat{G}_{w}$ resp. $\tilde{G}_{w}$ on $\underline{\hat{g}}_{w}$. If $V \in \Lambda$ and $W \in \mathfrak{C}^{\underline{w}}(L)$ then (notation as in 2.4.4)

$$
\left.\operatorname{Pr}\left(\operatorname{Ad}_{\underline{\underline{g}}_{w}}\left(\psi\left(g_{+}(V)\right) W\right)\right)=\operatorname{Ad}_{\underline{g}_{w}}\left(g_{+}(V)\right) W\right)=W .
$$

Hence

3.5 .3

$$
\operatorname{Ad}_{\underline{\underline{g}}_{w}}\left(\psi\left(g_{+}(V)\right) W\right)=W+\alpha\left(g_{+}(V), W\right) c,
$$

where $\alpha\left(g_{+}(V), W\right)$ describes the lifting of the action to $\underline{\hat{g}}_{w}$.

Lemma 3.5.4. Suppose $V \in \Lambda, W \in \mathfrak{C}^{w}(L)$. Then

$$
\Phi(V+W)=e^{-\frac{1}{2} \omega(V, W)+\gamma(V)+\alpha\left(g_{+}(V), W\right)} \Phi(W) .
$$

Proof.

$$
\begin{aligned}
\Phi(V+W) & =\left\langle\pi_{\lambda_{0}}(\exp (V+W)) v_{0}, v_{0}\right\rangle_{\lambda_{0}} \\
& =e^{-\frac{1}{2} \omega(V, W)}\left\langle\pi_{\lambda_{0}}(\exp (V)) \pi_{\lambda_{0}}(\exp (W)) v_{0}, v_{0}\right\rangle_{\lambda_{0}} \text { by } 3.5 .2 \\
& =e^{-\frac{1}{2} \omega(V, W)+\gamma(v)}\left\langle\pi_{\lambda_{0}}\left(\psi\left(g_{-}(V) g_{+}(V)\right)\right) \pi_{\lambda_{0}}(\exp W) v_{0}, v_{0}\right\rangle_{\lambda_{0}} \text { by 3.5.1. }
\end{aligned}
$$


If $X \in \underline{g}$, then $\left\langle X v_{0}, v_{0}\right\rangle_{\lambda_{0}}=0$ which follows from the contravariance of the form $\langle,\rangle_{\lambda_{0}}$ (cf. Garland [5], pp. 541-549) and the fact that $\underline{\hat{h}} \oplus \underline{\hat{n}}$ acts trivially on $\mathbb{C} v_{0}$. Since $\langle,\rangle_{\lambda_{0}}$ is positive definite (cf. Garland [5]) we conclude $X v_{0}=0$ for $X \in g$. Hence, if $g \in G$ then $g v_{0}=v_{0}$, since $G$ is connected. We also have $g v_{0}=v_{0}$ for $g \in \hat{V}_{w,+}$. Thus from the contravariance of the form $\langle,\rangle_{\lambda_{0}}$ we obtain

$$
\begin{aligned}
e^{-\frac{1}{2} \omega(V, W)+\gamma(V)}\langle & \left.\pi_{\lambda_{0}}\left(\psi\left(g_{-}(V) g_{+}(V)\right)\right) \pi_{\lambda_{0}}(\exp W) v_{0}, v_{0}\right\rangle_{\lambda_{0}} \\
= & e^{-\frac{1}{2} \omega(V, W)+\gamma(V)}\left\langle\pi_{\lambda_{0}}\left(\psi\left(g_{+}(V)\right)\right) \pi_{\lambda_{0}}(\exp W) v_{0}, v_{0}\right\rangle_{\lambda_{0}} \\
= & e^{-\frac{1}{2} \omega(V, W)+\gamma(V)}\left\langle\pi_{\lambda_{0}}\left(\exp \operatorname{Ad}_{\underline{g}_{w}}\left(\psi\left(g_{+}(V)\right)\right) W\right) v_{0}, v_{0}\right\rangle_{\lambda_{0}} \\
& \quad\left(G \hat{V}_{w,+} \text { acts by } 1 \text { on } \mathbb{C} v_{0}\right) \\
= & e^{-\frac{1}{2} \omega(V, W)+\gamma(W)+\alpha\left(g_{+}(V), W\right)}\left\langle\pi_{\lambda_{0}}(\exp W) v_{0}, v_{0}\right\rangle_{\lambda_{0}} \text { by } 3.5 .3 \\
= & e^{-\frac{1}{2} \omega(V, W)+\gamma(W)+\alpha\left(g_{+}(V), W\right)} \Phi(W) .
\end{aligned}
$$

Lemma 3.5.5. If $g_{1}, g_{1} \in \hat{G}_{w}, X \in \underline{\hat{g}}_{w}$ and $A d_{\underline{g}_{w}}\left(p r\left(g_{j}\right) X\right)=X, j=1,2$, then

$$
\alpha\left(g_{1} g_{2}, X\right)=\alpha\left(g_{1}, X\right)+\alpha\left(g_{2}, X\right)
$$

Proof.

$$
\operatorname{Ad}_{\underline{\hat{g}}_{w}}\left(g_{1} g_{2}\right) X=\operatorname{Ad}_{\underline{\underline{g}}_{w}}\left(\operatorname{pr}\left(g_{1} g_{2}\right) X\right)+\alpha\left(g_{1} g_{2}, X\right) c=X+\alpha\left(g_{1} g_{2}, X\right) c .
$$

Also,

$$
\operatorname{Ad}_{\underline{\underline{g}}_{w}}\left(g_{1} g_{2}\right) X=\operatorname{Ad}_{\underline{\underline{g}}_{w}}\left(g_{1}\right)\left(X+\alpha\left(g_{2}, X\right) c\right)=X+\alpha\left(g_{1}, X\right) c+\alpha\left(g_{2}, X\right) c .
$$

Suppose $g \in \hat{G}_{w}$ and $X \in \underline{\tilde{g}}_{w}$. Then

$$
\operatorname{Ad}_{\underline{\underline{g}}_{w}}(g) X=\operatorname{Ad}_{\underline{\tilde{g}}_{w}}(\operatorname{pr}(g)) X+\alpha(g, X) c
$$

Hence

$$
\begin{aligned}
\hat{B}^{e}\left(\operatorname{Ad}_{\underline{\underline{g}}_{w}}(g) X, d\right) & =\hat{B}^{e}\left(\operatorname{Ad}_{\underline{\underline{g}}_{w}}(\operatorname{pr}(g)) X+\alpha(g, X) c, d\right) \\
& =\hat{B}^{e}\left(\operatorname{Ad}_{\underline{g}_{w}}(\operatorname{pr}(g)) X, d\right)+\hat{B}^{e}(\alpha(g, X) c, d) \\
& =\alpha(g, X) .
\end{aligned}
$$


From the properties of $\hat{B}^{e}$ it follows

$$
\begin{aligned}
\hat{B}^{e}\left(\operatorname{Ad}_{\underline{\underline{g}}_{w}}(g) X, d\right) & =\hat{B}^{e}\left(X, \operatorname{Ad}_{\underline{\underline{g}}_{w}}(g)^{-1} d\right) \\
& =\hat{B}^{e}\left(X, g^{-1} d g\right) .
\end{aligned}
$$

Thus

$$
\alpha(g, X)=\hat{B}^{e}\left(X, g^{-1} d g\right)
$$

If $V \in \Lambda$ and $W \in \mathfrak{C}^{w}(L)$, then

$$
\begin{aligned}
\alpha\left(g_{+}(V), W\right) & =\hat{B}^{e}\left(W, g_{+}(V)^{-1} d g_{+}(V)\right) \\
& =\frac{1}{2 \pi i} \int_{0}^{2 \pi} B\left(W\left(e^{i \theta}\right),\left(g_{+}(V)\left(e^{i \theta}\right)\right)^{-1} \frac{\mathrm{d}}{\mathrm{d} \theta} g_{+}(V)\left(e^{i \theta}\right)\right) \mathrm{d} \theta
\end{aligned}
$$

which shows that the ambiguity in the definition of $g_{+}(V)$ by $a I, a I \in$ $G, a \in \mathbb{C}^{*}$, does not occur in the expression $\alpha\left(g_{+}(V), W\right)$. Since the map

$$
\Lambda \rightarrow C_{+}^{w}(L)_{/ \mathbb{C}^{*} I \cap G}
$$

given by $V \rightarrow g_{+}(V)$ is a homomorphism, we obtain from 3.5.5.

Lemma 3.5.6. If $U, V \in \Lambda$ and $W \in \mathfrak{C}^{w}(L)$, then

$$
\alpha\left(g_{+}(U+V), W\right)=\alpha\left(g_{+}(U), W\right)+\alpha\left(g_{+}(V), W\right) .
$$

Set $\delta(V, W)=\alpha\left(g_{+}(V), W\right)$. Then 3.5.4 can be restated as

$$
\Phi(V+W)=e^{-\frac{1}{2} \omega(V, W)+\gamma(V)+\delta(V, W)} \Phi(W) .
$$

Lemma 3.5.7. (1) If $V \in \mathfrak{C}_{+}^{w}(L), W \in \mathfrak{C}^{w}(L)$, then

$$
\Phi(V+W)=e^{\frac{1}{2} \omega(V, W)} \Phi(W) .
$$

(2) If $V \in \mathfrak{C}_{-}^{w}(L), W \in \mathfrak{C}^{w}(L)$, then

$$
\Phi(V+W)=e^{-\frac{1}{2} \omega(V, W)} \Phi(W) .
$$

Proof. (a) Since the cocycle $\omega$ vanishes on $\underline{\hat{g}}_{w,+}$ and on $\underline{\hat{g}}_{w,-}$ it follows $\gamma(V)=0$ for $V \in \mathfrak{C}_{+}^{w}(L)$ or $V \in \mathfrak{C}_{-}^{w}(L)$. 
(b) Suppose $V \in \mathfrak{C}_{+}^{w}(L)$. We assert $\delta(V, W)=\omega(V, W)$. Since $V \in$ $\mathfrak{C}_{+}^{w}(L)$ it follows $g_{+}(V)=e^{V}$. Moreover, $g_{+}(V)^{-1}=e^{-V}$ and $\frac{\mathrm{d}}{\mathrm{d} \theta} g_{+}(V)\left(e^{i \theta}\right)=$ $e^{V\left(e^{2 \theta}\right)} \frac{\mathrm{d}}{\mathrm{d} \theta} V\left(e^{i \theta}\right)$. Thus $\frac{\mathrm{d}}{\mathrm{d} \theta} V\left(e^{i \theta}\right)=\left(g_{+}(V)\left(e^{i \theta}\right)\right)^{-1} \frac{\mathrm{d}}{\mathrm{d} \theta} g_{+}(V)\left(e^{i \theta}\right)$. Hence

$$
\begin{aligned}
\omega(V, W) & =\frac{1}{2 \pi i} \int_{0}^{2 \pi} B\left(\frac{\mathrm{d}}{\mathrm{d} \theta} V\left(e^{i \theta}\right), W\left(e^{i \theta}\right)\right) \mathrm{d} \theta \\
& =\frac{1}{2 \pi i} \int_{0}^{2 \pi} B\left(\left(g_{+}(V)\left(e^{i \theta}\right)\right)^{-1} \frac{\mathrm{d}}{\mathrm{d} \theta} g_{+}(V)\left(e^{i \theta}\right), W\left(e^{i \theta}\right)\right) \mathrm{d} \theta \\
& =\delta(V, W) .
\end{aligned}
$$

(c) Since $\delta(V, W)=0$ for $V \in \mathfrak{C}_{-}^{w}(L)$, statement (2) follows.

For any $X \in \underline{\tilde{g}}_{w}$ with $X=X_{+}+X_{0}+X_{-}$, set

$$
\sigma(X)=\omega\left(X_{+}, X_{-}\right)
$$

Since for any $X, Y \in \underline{\tilde{g}}_{w},(X+Y)_{+}=X_{+}+Y_{+}$and $(X+Y)_{-}=X_{-}+Y_{-}$ it follows by integration by parts

$$
\begin{aligned}
\sigma(X+Y)-\sigma(X)-\sigma(Y) & =\omega\left(X_{+}+Y_{+}, X_{-}+Y_{-}\right)-\omega\left(X_{+}, X_{-}\right)-\omega\left(Y_{+}, Y_{-}\right) \\
& =\omega\left(X_{+}, Y_{-}\right)-\omega\left(X_{-}, Y_{+}\right) .
\end{aligned}
$$

Denote by

3.5.8.

$$
(X, Y)=\sigma(X+Y)-\sigma(X)-\sigma(Y) .
$$

Suppose $W \in \mathfrak{C}^{w}(L)$. Define the function $\Theta$ to be

3.5.9.

$$
\Theta(W)=e^{-\frac{1}{2} \sigma(W)} \Phi(W) .
$$

Lemma 3.5.10. Suppose $W \in \mathfrak{C}^{w}(L)$. If $V \in \mathfrak{C}_{+}^{w}(L)$ or $V \in \mathfrak{C}_{-}^{w}(L)$, then

$$
\Theta(V+W)=\Theta(W)
$$

Proof. (a) Suppose $W \in \mathfrak{C}^{w}(L)$ and $V \in \mathfrak{C}_{+}^{w}(L)$. Then

$$
\Phi(V+W)=e^{\frac{1}{2} \omega(V, W)} \Phi(W) .
$$

Thus

$$
\begin{aligned}
\Theta(V+W) & =e^{-\frac{1}{2} \sigma(V+W)} \Phi(V+W) \\
& =e^{-\frac{1}{2} \sigma(V+W)+\frac{1}{2} \omega(V, W)} \Phi(W) .
\end{aligned}
$$


We establish the formula by proving $\sigma(V+W)-\omega(V, W)=\sigma(W)$. Since the cocyle $\omega$ vanishes on $\underline{\tilde{g}}_{w,+}$ and $\frac{\mathrm{d}}{\mathrm{d} \theta} V_{+}\left(e^{i \theta}\right)=\frac{\mathrm{d}}{\mathrm{d} \theta} V\left(e^{i \theta}\right)$ it follows

$$
\begin{aligned}
\sigma(V+W)-\omega(V, W) & =\sigma(V+W)-\omega\left(V_{+}, W_{-}\right) \\
& =\omega\left(V_{+}+W_{+}, W_{-}\right)-\omega\left(V_{+}, W_{-}\right) \\
& =\omega\left(W_{+}, W_{-}\right) \\
& =\sigma(W) .
\end{aligned}
$$

(b) To prove the formula for $V \in \mathfrak{C}_{-}^{w}(L)$, one shows by a similar argument that $\sigma(V+W)+\omega(V, W)=\sigma(W)$.

Define for $V \in \Lambda / \mathfrak{C}_{-}^{w}(L)+\mathfrak{C}_{+}^{w}(L)$ and $W \in \mathfrak{C}^{w}(L) / \mathfrak{C}_{-}^{w}(L)+\mathfrak{C}_{+}^{w}(L)$

$$
\ell(W, V)=-\frac{1}{4 \pi i}[(V, W)+\omega(V, W)-2 \delta(V, W)]
$$

and

$$
J(V)=-\frac{1}{4 \pi i}[\sigma(V)-2 \gamma(V)]
$$

The definition of the function $\ell(W, V)$ shows that it is $\mathbb{C}$-linear in $W$, since $(V, W), \omega(V, W)$ and $\delta(V, W)$ are $\mathbb{C}$-linear in $W$.

We are now ready to prove one of the main results of this work which shows that the function $\Theta$ as defined in 3.5.9 is a theta function. This generalizes a result of Goodman-Wallach who showed in the case of $n=2$ and tridiagonal $L$ that the matrix entries of the standard representations are theta functions ( [8], pp. 211-213 - see also appendix).

Theorem 3.5.11.

Suppose $V \in \Lambda / \mathfrak{C}_{-}^{w}(L)+\mathfrak{C}_{+}^{w}(L)$ and $W \in \mathfrak{C}^{w}(L) / \mathfrak{C}_{-}^{w}(L)+\mathfrak{C}_{+}^{w}(L)$. Then

$$
\Theta(V+W)=e^{2 \pi i[\ell(W, V)+J(V)]} \Theta(W) .
$$

Proof. This is the functional equation required in Lang's definition of a theta function ([13], Chapter 4, (1.2)). 
(by 3.5.9)

$$
\Theta(V+W)=e^{-\frac{1}{2} \sigma(V+W)} \Phi(V+W)
$$

(by 3.5 .4 )

$$
=e^{-\frac{1}{2}[\sigma(V+W)+\omega(V, W)]+\delta(V, W)+\gamma(V)} \Phi(W)
$$

(by 3.5 .8 )

$$
=e^{-\frac{1}{2}[\sigma(V)+\sigma(W)+(V, W)+\omega(V, W)]+\delta(V, W)+\gamma(V)} \Phi(W)
$$

(by 3.5 .9 )

$$
\begin{aligned}
& =e^{-\frac{1}{2}[(V, W)+\omega(V, W)-2 \delta(V, W)]-\frac{1}{2}[\sigma(V)-2 \gamma(V)]} \Theta(W) \\
& =e^{2 \pi i[\ell(V, W)+J(V)]} \Theta(W) .
\end{aligned}
$$

\section{Appendix. The Case $\widehat{S L}(2, \mathbb{R})$.}

This section contains a calculation of the solution of the generalized periodic Toda lattice in the case of $\widehat{S I}(2, \mathbb{R})$. It was shown by Goodman and Wallach that the solutions are given in terms of representative functions of standard modules. The representative functions are expressed in terms of the original Jacobi theta function. The calculation is taken from $([\mathbf{8}]$, p. 211-213 - see also references therein).

A) Let $H$ denote the periodic Toda lattice system for $\widehat{S L}(2, \mathbb{R})$ :

$$
H=\frac{1}{4} \cdot p_{1}^{2}+\frac{1}{2}\left(e^{-2 q_{0}}+e^{-2 q_{1}}\right)
$$

with $q_{0}=\gamma-q_{1}$ for some constant $\gamma$,

$$
\begin{aligned}
& \dot{q_{1}}=-\frac{\partial H}{\partial p_{1}}=-\frac{1}{2} p_{1}, \quad \dot{q}_{0}=-\dot{q}_{1}, \\
& \dot{p_{1}}=\frac{\partial H}{\partial q_{1}}=e^{-2 q_{0}}-e^{-2 q_{1}}
\end{aligned}
$$

and

$$
q_{0}(0)=-\log \left(\frac{1+k}{\sqrt{2}}\right), q_{1}(0)=-\log \left(\frac{1-k}{\sqrt{2}}\right), p_{1}(0)=0 .
$$

Here $k$ denotes a parameter in the range $-1<k<1$. Assume that $L$ is as follows:

$$
L(z)=\left[\begin{array}{cc}
0 & c_{0} \cdot z^{-1}+c_{1} \\
c_{0} \cdot z+c_{1} & 0
\end{array}\right]
$$


with $c_{0}=\frac{1}{2}(1+k), c_{1}=\frac{1}{2}(1-k)$.

Let $\phi_{i}(t)$ be defined by

$$
\phi_{i}(t)=\left\langle\pi_{\lambda_{i}} \exp (-t L) v_{i}, v_{i}\right\rangle_{\lambda_{i}}
$$

for $i=0,1$.

The solution of the periodic Toda lattice is given by

$$
q_{1}(t)=q_{1}(0)+\log \left\{\frac{\phi_{1}(t)}{\phi_{0}(t)}\right\} .
$$

B) Define $x, y$ and $z$ as follows:

$$
x=2^{-\frac{1}{2}}\left(e^{-q_{0}}-e^{-q_{1}}\right), y=\frac{1}{2} \cdot p_{1}, z=2^{-\frac{1}{2}}\left(e^{-q_{0}}+e^{-q_{1}}\right) .
$$

Then $x, y$ and $z$ satisfy the system of bilinear differential equations

$$
\dot{x}=-y \cdot z, \dot{y}=x \cdot z, \dot{z}=-x \cdot y .
$$

Given the above initial condition the solution of $x, y$ and $z$ are given in terms of the Jacobi elliptic functions as

$$
x=k c n(t, k), y=k \operatorname{sn}(t, k), z=d n(t, k) .
$$

The coordinate functions of the Hamilitonian are then expressed as

$$
\begin{aligned}
& q_{1}(t)=q_{1}(0)+\log \left\{\frac{d n(t, k)+k c n(t, k)}{1+k}\right\} \\
& p_{1}(t)=2 k \operatorname{sn}(t, k) .
\end{aligned}
$$

C) For the representative functions we have

$$
\log \left\{\frac{\phi_{1}(t)}{\phi_{0}(t)}\right\}=\frac{d n(t, k)+k c n(t, k)}{1+k}=\frac{d n(t, k)-k c n(t, k)}{1-k}
$$

The representative functions $\phi_{i}(t)$ also satisfy the differential equation

$$
c_{i}^{2} \phi_{i}(t)=\phi_{i}^{\prime \prime}(t) \phi_{i}(t)-\left[\phi_{i}(t)\right]^{2}
$$

from which we obtain

$$
\begin{aligned}
& 2\left(\log \phi_{0}\right)^{\prime \prime}=d n^{2}+k \cdot d n \cdot c n+2\left(k^{2}-1\right) \\
& 2\left(\log \phi_{1}\right)^{\prime \prime}=d n^{2}-k \cdot d n \cdot c n+2\left(k^{2}-1\right) .
\end{aligned}
$$


These equations can be integrated using complete elliptic integrals of the first and second kind with modulus $k$ to yield:

$$
\phi_{0}(t)^{2}=\frac{\theta_{4}\left(\frac{\pi t}{2 K}, q\right)}{\theta_{4}(0, q)} \cdot \frac{d n(t, k)-k c n(t, k)}{1-k} e^{2 v t^{2}}
$$

where $q, 0 \leq q<1$, is defined implicitely by

$$
1-k^{2}=\prod_{n=1}^{\infty}\left\{\frac{1-q^{2 n-1}}{1+q^{2 n-1}}\right\}^{8},
$$

and $E, K, v$ are given by

$$
\begin{aligned}
K & =\int_{0}^{\frac{\pi}{2}}\left(1-k^{2} \sin ^{2} \theta\right)^{-\frac{1}{2}} d \theta \\
E & =\int_{0}^{\frac{\pi}{2}}\left(1-k^{2} \sin ^{2} \theta\right)^{\frac{1}{2}} d \theta \\
v & =\frac{k^{2}-1}{8}+\frac{E}{4 K}
\end{aligned}
$$

Similarly one obtains for $\phi_{1}(t)^{2}$

$$
\phi_{1}(t)^{2}=\frac{\theta_{4}\left(\frac{\pi t}{2 K}, q\right)}{\theta_{4}(0, q)} \cdot \frac{d n(t, k)+k c n(t, k)}{1+k} e^{2 v t^{2}} .
$$

From infinite product expansions for

$$
d n(t, k) \pm k c n(t, k)
$$

and from Jacobi's infinite product expansions of theta functions we obtain explicit expressions for $\phi_{0}(t), \phi_{1}(t)$ in terms of theta functions. The formulas for $\phi_{i}(t)$ are given as

$$
\begin{aligned}
& \phi_{0}(t)=e^{v t^{2}} \frac{\theta_{4}\left(\frac{u}{2}, q^{\frac{1}{2}}\right)}{\theta_{4}\left(0, q^{\frac{1}{2}}\right)} \\
& \phi_{1}(t)=e^{v t^{2}} \frac{\theta_{3}\left(\frac{u}{2}, q^{\frac{1}{2}}\right)}{\theta_{3}\left(0, q^{\frac{1}{2}}\right)}
\end{aligned}
$$

with $u=\frac{\pi t}{2 K}$.

\section{References}

[1] M. Adler and P. van Moerbeke, Completely integrable systems, Euclidean Lie algebras and curves, Adv. Math., 38 (1980), 267-317. 
[2] M. Adler and P. van Moerbeke, Linearization of Hamiltonian systems, Jacobion varieties and representation theory, Adv. Math., 38 (1980), 318-379.

[3] L. Bers, Riemann surfaces, Lectures, New York University 1957-58.

[4] H.M. Farkas and I. Kra, Riemann surfaces, Springer Verlag, New York 1980.

[5] H. Garland, The arithmetic theory of loop algebras, J. Algebra, 53 (1978), 480-551.

[6] R. Goodman and N.R. Wallach, Classical and quantum-mechanical systems of Toda lattice type I, Commun. Math. Phys., 83 (1982), 355-386.

[7] R. Goodman and N.R. Wallach, Structure and unitary cocycle representations of loop groups and the group of diffeomorphisms of the circle, J. Reine Angew. Math., 347 (1984), 69-113.

[8] R. Goodman and N.R. Wallach, Classical and quantum-mechanical systems of Toda lattice type II, Commun. Math. Phys., 94 (1984), 177-217.

[9] R. Goodman and N.R. Wallach, Classical and quantum-mechanical systems of Toda lattice type III, Commun. Math. Phys., 105 (1986), 473-509.

[10] P. Griffiths and J. Adams, Topics in algebraic and analytic geometry, Princeton University Press, Princeton 1974.

[11] R.C. Gunning, Lectures on Riemann surfaces, Princeton University Press, Princeton 1966.

[12] R. Hartshorne, Algebraic Geometry, Springer Verlag, New York 1977.

[13] S. Lang, Introduction to algebraic and abelian functions, Addison - Wesley, Reading 1972.

[14] P. van Moerbeke and D. Mumford, The spectrum of difference operators and algebraic curves, Acta Math., 143 (1979), 93-154.

[15] J. Moser, Geometry of quadrics and spectral theory, The Chern Symposium 1979: Proc. of the International Symposium on Differential Geometry, Berkeley, CA 1979.

[16] D. Mumford, Algebraic geometry I Complex Projective Varieties, Springer Verlag, New York 1976.

[17] D. Mumford, Tata lectures on theta I, Birkhäuser, Boston 1983.

[18] D. Mumford, Tata lectures on theta II, Birkhäuser, Boston 1983.

[19] A. Pressley and G. Segal, Loop groups and their representations, Oxford University Press, Oxford.

[20] A.G. Reyman and M.A. Semenov-Tian-Shansky, Reduction of Hamiltonian systems, affine Lie algebras and Lax equations, Invent. Math., 54 (1979), 81-100.

[21] A.G. Reyman and M.A. Semenov-Tian-Shansky, Reduction of Hamiltonian systems, affine Lie algebras and Lax equations, II, Invent. Math., 63 (1981), 423-432.

[22] G.B. Segal and G. Wilson, Loop groups and equations of KdV type, Pub. Math. I. M. E. S., 61 (1985), 5-65.

[23] J.-P. Serre, Géométrie algébrique et géométrie analytique, Annales Inst. Fourier, 6 (1955-1956), 1-42.

[24] J.-P. Serre, Groupes algébrique et corps de classes, Hermann, Paris 1959.

[25] B.L. van der Waerden, Algebra I, Springer Verlag, Berlin 1936.

[26] N.R. Wallach, Harmonic analysis on homogeneous spaces, Marcel Dekker, New York 1973. 
[27] N.R. Wallach, Lecture notes, Rutgers University 1987.

Received January 6, 1994 and revised April 15, 1994.

IM HIRSCHMORGEN 14

69181 LEIMEN

Federal Republic of Germany

E-mail address: POLYCARPOU@EMBL-Heidelberg.DE 


\title{
PACIFIC JOURNAL OF MATHEMATICS
}

\author{
Founded in 1951 by
}

\author{
$\begin{array}{ll}\text { E. F. Beckenbach (1906-1982) } & \text { F. Wolf (1904-1989) }\end{array}$
}

\section{EDITORS}

Sun-Yung A. Chang (Managing Editor) Robert Finn University of California

Los Angeles, CA 90095-1555

pacific@math.ucla.edu

\section{F. Michael Christ}

University of California

Los Angeles, CA 90095-1555

christ@math.ucla.edu

Nicholas Ercolani

University of Arizona

Tucson, AZ 85721

ercolani@math.arizona.edu
Stanford University

Stanford, CA 94305

finn@gauss.stanford.edu

Steven Kerckhoff

Stanford University

Stanford, CA 94305

spk@gauss.stanford.edu

Martin Scharlemann

University of California

Santa Barbara, CA 93106

mgscharl@math.ucsb.edu

\section{Gang Tian}

Massachusettes Institute of Technology

Cambridge, MA 02139

tian@math.mit.edu

\section{S. Varadarajan University of California Los Angeles, CA 90095-1555 vsv@math.ucla.edu \\ Dan Voiculescu \\ University of California \\ Berkeley, CA 94720 \\ dvv@math.berkeley.edu}

\section{SUPPORTING INSTITUTIONS}

ACADEMIA SINICA, TAIPEI

CALIF. INST. OF TECHNOLOGY

CHINESE UNIV. OF HONG KONG

HONG KONG UNIV. OF SCI. \& TECH.

KEIO UNIVERSITY

MACQUARIE UNIVERSITY

MATH. SCI. RESEARCH INSTITUTE

NEW MEXICO STATE UNIV.

OREGON STATE UNIV.

PEKING UNIVERSITY

RITSUMEIKAN UNIVERSITY

STANFORD UNIVERSITY

\author{
TOKYO INSTITUTE OF TECHNOLOGY \\ UNIVERSIDAD DE LOS ANDES \\ UNIV. OF ARIZONA \\ UNIV. OF BRITISH COLUMBIA \\ UNIV. OF CALIF., BERKELEY \\ UNIV. OF CALIF., DAVIS \\ UNIV. OF CALIF., IRVINE \\ UNIV. OF CALIF., LOS ANGELES \\ UNIV. OF CALIF., RIVERSIDE \\ UNIV. OF CALIF., SAN DIEGO \\ UNIV. OF CALIF., SANTA BARBARA
}

UNIV. OF CALIF., SANTA CRUZ

UNIV. OF HAWAII

UNIV. OF MELBOURNE

UNIV. OF MONTANA

UNIV. NACIONAL AUTONOMA DE MEXICO

UNIV. OF NEVADA, RENO

UNIV. OF OREGON

UNIV. OF SOUTHERN CALIFORNIA

UNIV. OF UTAH

UNIV. OF WASHINGTON

WASHINGTON STATE UNIVERSITY

The supporting Institutions listed above contribute to the cost of publication of this Journal, but they are not owners or publishers and have no responsibility for its contents or policies.

Manuscripts must be prepared in accordance with the instructions provided on the inside back cover.

The table of contents and the abstracts of the papers in the current issue, as well as other information about the Pacific Journal of Mathematics, may be found on the Internet at http://www.math.uci.edu/pjm.html.

The Pacific Journal of Mathematics (ISSN 0030-8730) is published monthly except for July and August. Regular subscription rate: $\$ 245.00$ a year (10 issues). Special rate: $\$ 123.00$ a year to individual members of supporting institutions.

Subscriptions, back issues published within the last three years and changes of subscribers address should be sent to Pacific Journal of Mathematics, P.O. Box 4163, Berkeley, CA 94704-0163, U.S.A. Prior back issues are obtainable from Kraus Periodicals Co., Route 100, Millwood, NY 10546.

The Pacific Journal of Mathematics at the University of California, c/o Department of Mathematics, 981 Evans Hall, Berkeley, CA 94720 (ISSN 0030-8730) is published monthly except for July and August. Second-class postage paid at Berkeley, CA 94704, and additional mailing offices. POSTMASTER: send address changes to Pacific Journal of Mathematics, P.O. Box 6143, Berkeley, CA 94704-0163.

\section{PUBLISHED BY PACIFIC JOURNAL OF MATHEMATICS at University of California,} Berkeley, CA 94720, A NON-PROFIT CORPORATION

This publication was typeset using AMS-LATEX,

the American Mathematical Society's TEX macro system.

Copyright (C) 1995 by Pacific Journal of Mathematics 


\section{PACIFIC JOURNAL OF MATHEMATICS}

\section{Volume $176 \quad$ No. $2 \quad$ December 1996}

One remark on polynomials in two variables

ENRIQUe ARTAl BARTOLO and PIERRETTE CASSOU-NOGUÈS

Divergence of the normalization for real Lagrangian surfaces near complex tangents XIANGHONG GONG

Classification of the stable homotopy types of stunted lens spaces for an odd prime JESUS GONZALEZ

Plancherel formulae for non-symmetric polar homogeneous spaces

JING-SONG HUANG

A uniqueness theorem for the minimal surface equation

JENN-FANG HWANG

Differential Galois groups of confluent generalized hypergeometric equations: an approach

using Stokes multipliers

Claudine Mitschi

Oscillatory theorem and pendent liquid drops

KIMIAKI NARUKAWA and TAKASHI SUZUKI

Local and global plurisubharmonic defining functions

ALAN NOELL

Specializations and a local homeomorphism theorem for real Riemann surfaces of rings

M. J. DE LA PUENTE

Eigenvalue comparisons in graph theory

GREGORY T. QUENELL

Applications of loop groups and standard modules to Jacobians and theta functions of isospectral curves

WILLI SCHWARZ

Bridged extremal distance and maximal capacity

ROBERT E. THURMAN

Imbedding and multiplier theorems for discrete Littlewood-Paley spaces

IGOR E. VERBITSKY

On constrained extrema

THOMAS VOGEL

Heat flow of equivariant harmonic maps from $\mathbb{B}^{3}$ into $\mathbb{C P} \mathbb{P}^{2}$

YUANLONG XIN

Proof of Longuerre's theorem and its extensions by the method of polar coordinates

ZHIHONG YU

Correction to: "Special generating sets of purely inseparable extension fields of unbounded exponent"

BONIFACE IHEMOTUONYE EKE 